\title{
Stability and robustness issues in numerical modeling of material failure with the strong discontinuity approach
}

\author{
J. Oliver *, A.E. Huespe, S. Blanco, D.L. Linero \\ E.T.S. d'Enginyers de Camins, Canals i Ports, Technical University of Catalonia (UPC), Campus Nord UPC, Mòdul C-1, \\ clJordi Girona 1-3, 08034 Barcelona, Spain
}

Received 29 September 2004; received in revised form 13 April 2005; accepted 15 April 2005

\begin{abstract}
Robustness and stability of the Continuum Strong Discontinuity Approach (CSDA) to material failure are addressed. After identification of lack of symmetry of the finite element formulation and material softening in the constitutive model as possible causes of loss of robustness, two remedies are proposed: (1) the use of an specific symmetric version of the elementary enriched (E-FEM) finite element with embedded discontinuities and (2) a new implicit-explicit integration of the internal variable, in the constitutive model, which renders the tangent constitutive algorithmic operator positive definite and constant. The combination of both developments leads to finite element formulations with constant, in the time step, and non-singular tangent structural stiffness, allowing dramatic improvements in terms of robustness and computational costs. After assessing the convergence and stability properties of the new strategies, three-dimensional numerical simulations of failure problems illustrate the performance of the proposed procedures.
\end{abstract}

(C) 2005 Elsevier B.V. All rights reserved.

Keywords: Computational material failure; Strong discontinuities; Localization; Implicit integration; Explicit integration

\section{Introduction}

During the last years, the Strong Discontinuity Approach (SDA) has appeared as a promising tool to model material failure in quasi brittle materials $[1,4,8-10,23,24,35]$. As an specific branch, the Continuum Strong Discontinuity Approach (CSDA) offers some additional features already presented in a number of works [22,27-31]. In essence, they allow capturing both the volume and surface dissipative effects, taking place during the fracturing process, using a standard continuum (i.e. stress vs. strain) format for the constitutive model. Some applications of the CSDA to modelling fracture of concrete have been recently reported [33]. Although those previous works state the ability of the CSDA to deal with local material failure and fracture propagation, in two-dimensional and simple three-dimensional cases, its applicability to model the structural collapse and the ultimate loading capacity of actual three-dimensional structures at acceptable computational costs was still limited. The main reason for this is the appearance of instabilities, inherent to the numerical procedure, which translate into some lack of robustness of the computational tool. This demands the use of skilful solution procedures to trace the equilibrium response (for instance, specific arc-length methods and automatic time stepping [29]), which translate into large computational costs.

Therefore, some new developments devoted to increase the robustness of computational procedures in the context of computational material failure and, in particular, of the CSDA seem to be lacking. This is the topic of this paper; recent developments of the authors in the context of the CSDA, addressed to increase the stability and robustness of the

\footnotetext{
* Corresponding author. Fax: +34 934011048.

E-mail addresses: xavier.oliver@upc.edu, xavier.oliver@upc.es (J. Oliver).
} 
numerical simulations and to decrease the computational cost of material failure analyzes, are presented. First, in Section 1.1 an overview of the foundations of the CSDA is presented. Then, in subsequent sections, two new developments concerning numerical aspects of that methodology are presented: (i) a specific implementation of a symmetric finite element with embedded discontinuities (E-FEM), and (ii) a new implicit/explicit integration algorithm for the constitutive model. The inclusion of both of them, translate into very large improvements in terms of the robustness as well as of the computational costs, which, actually, open the way to the use of the CSDA in the material failure analysis and structural collapse modelling of three-dimensional structures. Finally, in the last sections of the paper, applications to representative examples are presented.

\subsection{Basic aspects of the continuum-strong discontinuity approach}

The CSDA grounds on the classical continuum mechanics by generalizing the admissible displacement space and introducing a discontinuous field into the problem. The resulting kinematics, termed strong discontinuity kinematics, requires a reinterpretation of the constitutive model to make it capable of dealing with the unbounded strains emerging from those discontinuous displacement fields. In fact, from physical requirements the constitutive model should furnish bounded stresses even for unbounded strains. This can be achieved through the redefinition of only one parameter: the softening modulus, which has to be regularized in points where unbounded strains take place. The rest of ingredients and features of the continuum constitutive model remain unmodified.

Therefore, we can synthesize the computational CSDA methodology by the following four points.

1.1.1. Strong discontinuity kinematics

An admissible displacement field, $\mathbf{u}(\mathbf{x})$, exhibiting displacement discontinuities can be described by (see Fig. 1):

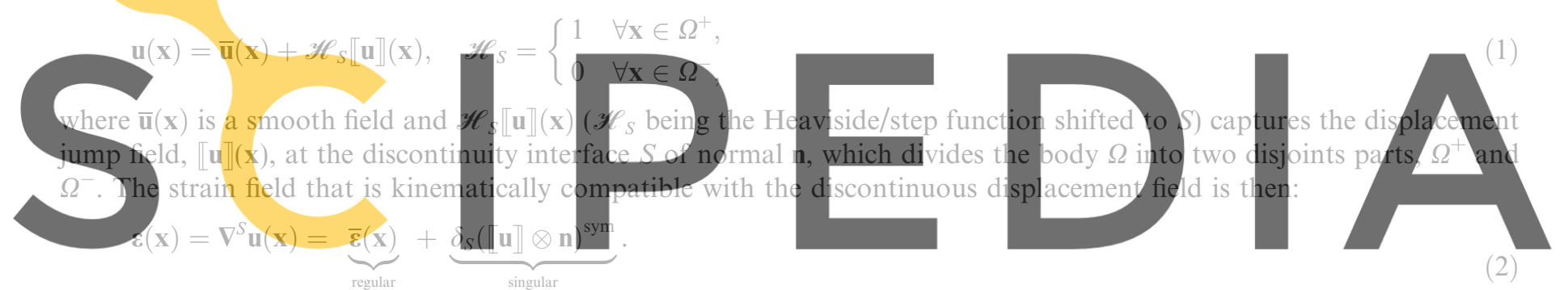

Register for free at https//www.scipedia.com to download the version without the watermark For computational purposes the surface Dirac's delta function $\delta_{S}$ in Eq. (2) is regularized in terms of a, very small, regularization parameter $k$ and a collocation function $\mu_{S}(\mathbf{x})$ on the discontinuity interface $S$. Then, the regularized version of the strain field reads:

$$
\boldsymbol{\varepsilon}(\mathbf{x})=\nabla^{S} \mathbf{u}(\mathbf{x})=\underbrace{\overline{\mathbf{\varepsilon}}(\mathbf{x})}_{\substack{\text { regular } \\
\text { (bounded) }}}+\underbrace{\frac{\mu_{S}}{k}(\llbracket \mathbf{u} \rrbracket \otimes \mathbf{n})^{\text {sym }}}_{\begin{array}{c}
\text { singular } \\
\text { (unbounded as } k \rightarrow 0)
\end{array}}, \quad \mu_{S}(\mathbf{x})= \begin{cases}1 & \text { for } \mathbf{x} \in S, \\
0 & \text { for } \mathbf{x} \in \Omega / S .\end{cases}
$$
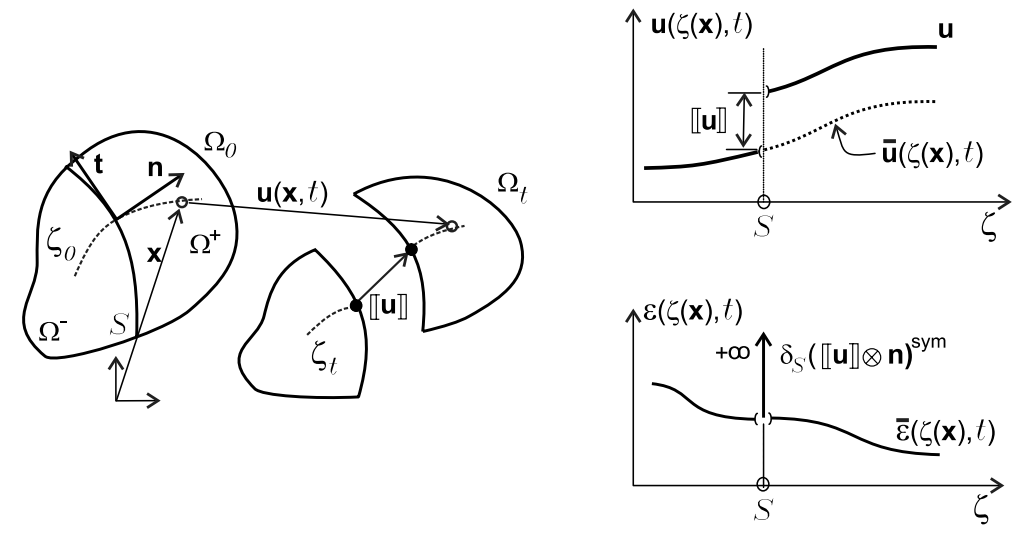

Fig. 1. Strong discontinuity kinematics. 


\subsubsection{Continuum constitutive model with regularized strain softening}

The choice of the continuum (i.e. stress vs. strain) constitutive model must be done in accordance with the phenomenological behaviour of the material whose failure is being reproduced. However, there is no intrinsic limitation on the type or family of that constitutive model in the context of the CSDA: any non-linear (dissipative) constitutive model equipped with strain softening can be considered. For the sake of covering a wide range of quasi-brittle materials, in the remaining of this paper two families of those continuum models will be considered: (1) isotropic continuum damage models and (2) elasto-plastic models with strain softening. The main ingredients of both models can be described as follows $[26,42]$ :

\begin{tabular}{|c|c|c|c|}
\hline & Isotropic continuum damage model & $\begin{array}{l}\text { Elasto-plastic model with } \\
\text { isotropic softening }\end{array}$ & \\
\hline Free energy & $\begin{array}{l}\varphi(\boldsymbol{\varepsilon}, r)=(1-d) \varphi_{0}, \quad \varphi_{0}=\frac{1}{2} \boldsymbol{\varepsilon}: \mathbf{C}^{e}: \boldsymbol{\varepsilon} \\
d(r)=1-q(r) / r\end{array}$ & $\begin{array}{l}\varphi\left(\boldsymbol{\varepsilon}^{e}, r\right)=\frac{1}{2} \boldsymbol{\varepsilon}^{e}: \mathbf{C}^{e}: \boldsymbol{\varepsilon}^{e}+\varphi^{p}(r) \\
\boldsymbol{\varepsilon}=\boldsymbol{\varepsilon}^{e}+\boldsymbol{\varepsilon}^{p}\end{array}$ & (4) \\
\hline Constitutive equation & $\boldsymbol{\sigma}=(1-d) \mathbf{C}^{e}: \boldsymbol{\varepsilon}=\frac{q}{r} \underbrace{\mathbf{C}^{e}: \boldsymbol{\varepsilon}}_{\bar{\sigma}}=\frac{q}{r} \overline{\boldsymbol{\sigma}}$ & $\boldsymbol{\sigma}=\mathbf{C}^{e}: \boldsymbol{\varepsilon}^{e}=\mathbf{C}^{e}:\left(\boldsymbol{\varepsilon}-\boldsymbol{\varepsilon}^{p}\right)$ & (6) \\
\hline
\end{tabular}
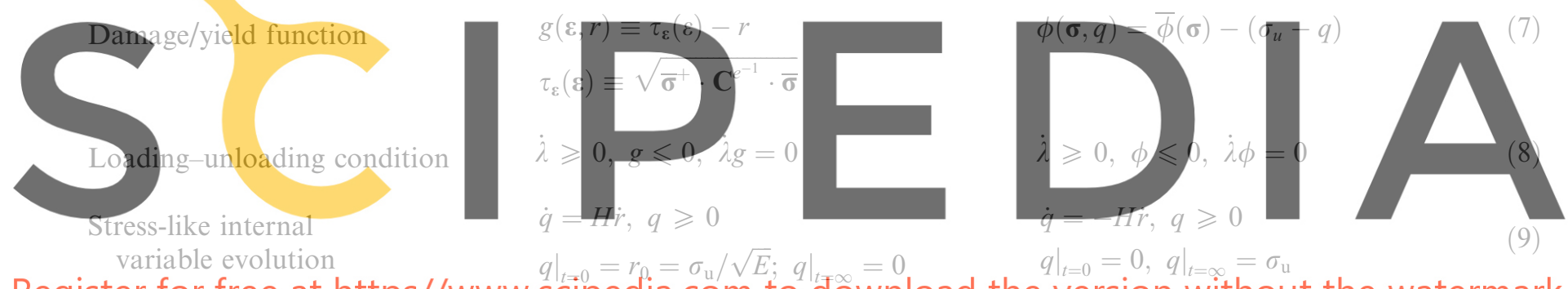

Register for free at https//www.scipedia.com to download the
Constitutive $\quad \dot{\sigma}=\mathrm{C}^{\tan }: \dot{\varepsilon}$,

tangent operator

$$
\mathbf{C}^{\tan }=\left\{\begin{array}{l}
\mathbf{C}^{\mathrm{u}} \equiv(1-d) \mathbf{C}^{e}=\frac{q}{r} \mathbf{C}^{e} \\
\mathbf{C}^{l} \equiv \frac{q}{r} \mathbf{C}^{e}-\frac{q-H r}{r^{3}} \overline{\boldsymbol{\sigma}}^{+} \otimes \overline{\boldsymbol{\sigma}}
\end{array}\right.
$$

$\dot{\sigma}=\mathrm{C}^{\mathrm{tan}}: \dot{\varepsilon}$,

$$
\mathbf{C}^{\tan }=\left\{\begin{array}{l}
\mathbf{C}^{\mathrm{u}} \equiv \mathbf{C}^{e} \\
\mathbf{C}^{l} \equiv \mathbf{C}^{e}-\frac{\mathbf{C}^{e}: \boldsymbol{\xi} \otimes \mathbf{C}^{e}: \boldsymbol{\xi}}{\boldsymbol{\xi}: \mathbf{C}^{e}: \boldsymbol{\xi}+H} \\
\boldsymbol{\xi}=\partial_{\boldsymbol{\sigma}} \phi(\boldsymbol{\sigma}, q)
\end{array}\right.
$$

where $\varphi(\boldsymbol{\varepsilon}, r)$ is the free energy, depending on the strain tensor $\boldsymbol{\varepsilon}$ (or the elastic part $\boldsymbol{\varepsilon}^{e}$ in the plastic model) and the internal variable $r$. The term $\varphi_{0}$ in the damage model is the elastic strain energy for the elastic (undamaged) material. $\mathbf{C}^{e}=\bar{\lambda}(\mathbf{1} \otimes \mathbf{1})+2 \mu \mathbf{I}$ is the elastic constitutive tensor, where $\bar{\lambda}$ and $\mu$ are the Lame's parameters and $\mathbf{1}$ and $\mathbf{I}$ are the identity tensors of second and fourth order, respectively. In Eq. (6), $\overline{\boldsymbol{\sigma}}=\mathbf{C}^{e}: \boldsymbol{\varepsilon}$ is the effective stress. Its positive counterpart is then defined as

$$
\overline{\boldsymbol{\sigma}}^{+}=\sum_{i=1}^{i=3}\left\langle\bar{\sigma}_{i}\right\rangle \mathbf{p}_{i} \otimes \mathbf{p}_{i},
$$

where $\left\langle\bar{\sigma}_{i}\right\rangle$ stands for the positive part (Mac Auley bracket) of the ith principal effective stress $\bar{\sigma}_{i}\left(\left\langle\bar{\sigma}_{i}\right\rangle=\bar{\sigma}_{i}\right.$ for $\bar{\sigma}_{i}>0$ and $\left\langle\bar{\sigma}_{i}\right\rangle=0$ for $\left.\bar{\sigma}_{i}<0\right)$ and $\mathbf{p}_{i}$ stands for the $i$ th stress eigenvector. The initial elastic domain in the damage model is defined as $E_{\sigma}^{0}:=\left\{\boldsymbol{\sigma}, \sqrt{\boldsymbol{\sigma}^{+} \cdot \mathbf{C}^{e^{-1}} \cdot \boldsymbol{\sigma}}<r_{0}\right\}$ and, therefore, it is unbounded for compressive stress states $\left(\boldsymbol{\sigma}^{+}=\mathbf{0}\right)$ so that damage becomes only associated to tensile stress states as it is usual for modelling tensile failure in quasi brittle materials like concrete.

The actual stresses, $\boldsymbol{\sigma}$, and the stress-like variable, $q$, are determined via the state Eqs. (6) and (9). The last equation defines the softening law in terms of the continuum softening parameter $H(r) \leqslant 0$ which may be either constant or an increasing function of $r, \sigma_{\mathrm{u}}$ and $E$ are, respectively, the tensile strength (yield stress) and the Young's modulus. Finally, 
Eq. (10) is the rate constitutive law in terms of the tangent constitutive operator $\mathbf{C}^{\text {tan }}$, which changes for loading $\left(\mathbf{C}^{\mathrm{l}}\right)$ and unloading $\left(\mathbf{C}^{\mathrm{u}}\right)$ processes.

The constitutive model should be adapted to return bounded stresses when the singular (unbounded) strain field (2) is introduced into the standard continuum context. This regularization is reached by a reinterpretation of the continuum softening modulus, $H$, in Eq. (9), which is expressed, in the distributional sense [41], in terms of a discrete softening modulus $\bar{H}$, considered a material property available in terms of the mechanical and fracturing properties of the material (peak stress $\sigma_{\mathrm{u}}$, Young's modulus $E$, and fracture energy $G_{\mathrm{f}}$, see $[25,30]$ for additional details).

$$
\begin{aligned}
& \frac{1}{H}=\delta_{S} \frac{1}{\bar{H}}, H=k \bar{H}, \\
& \left\{\begin{array}{l}
\bar{H}=\frac{1}{2} \frac{\sigma_{\mathrm{u}}^{2}}{E G_{\mathrm{f}}} \quad \text { (continuum damage) }, \\
\bar{H}=\frac{1}{2} \frac{\sigma_{\mathrm{u}}^{2}}{G_{\mathrm{f}}} \quad \text { (elasto-plasticity) } .
\end{array}\right.
\end{aligned}
$$

\subsubsection{Boundary value problem in a strong discontinuity setting}

The rate form of the BVP of a solid $\Omega$ with boundary $\partial \Omega=\Gamma_{\mathrm{u}} \cup \Gamma_{\sigma}$ (where $\Gamma_{\mathrm{u}}$ and $\Gamma_{\sigma}$ stand, respectively, for the boundaries with prescribed displacements and tractions) and outward normal $v$, experiencing a strong discontinuity of the displacement field $\mathbf{u}(\mathbf{x}, t)$ in a failure surface $S$ with normal $\mathbf{n}$ (see Fig. 1), in the time interval of interest $[0, T]$, can be written as follows:

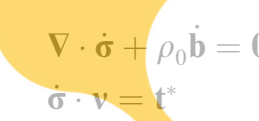

$$
\begin{aligned}
& \forall(\mathbf{x}, t) \in \Omega \times[0, T] \quad \text { (momentum balance), } \\
& \forall(\mathbf{x}, t) \in \Gamma_{\sigma} \times[0, T] \quad \text { (prescribed tractions), }
\end{aligned}
$$

(b)
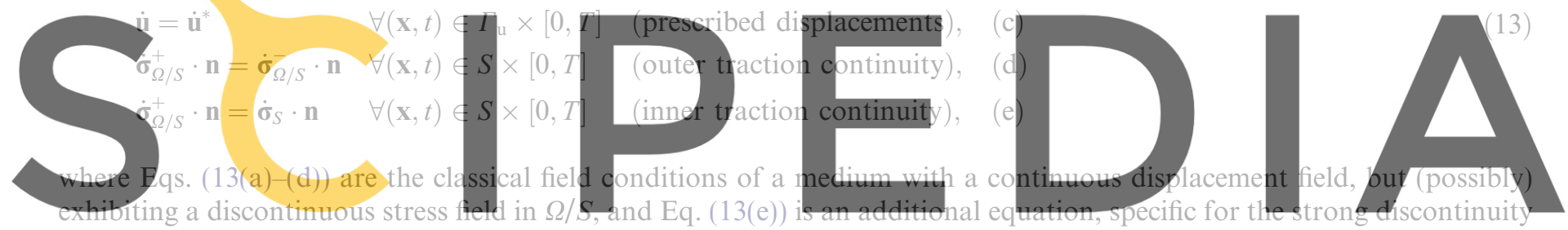

problem, stating the continuity of the traction field across the failure interface $S$. Register for free at https//www.scipedia.com to download the version without the watermark

Finite elements with embedded discontinuities have become a natural numerical ingredient in modeling material failure $[2,3,24,40]$ and are a fundamental tool within the CSDA. They consist of the addition, to the standard deformation modes of the basic element, of enriching deformation modes incorporating discontinuous displacement fields. The different families of those elements developed so far could be classified into the following categories:

- Nodal enrichment (X-FEM): The enriching modes have a nodal support i.e.: the set of elements sharing a specific node. The ones developed up to date are based on the X-FEM method [6,19], which, in turn, lies on the use of the partition of unity concept [6]. The additional degrees of freedom are attached to those regular nodes belonging to any element crossed by the discontinuity. Therefore, they cannot be condensed at the elemental level.

- Elemental enrichment (E-FEM): The enriching modes have an elemental support. The additional degrees of freedom representing the elemental displacement jump are attached to those elements crossed by the discontinuity [16]. They can be condensed at the elemental level and, thus, they do not substantially contribute to enlarge the computational cost of the analysis.

Although advantages and disadvantages in both families have been reported [16,29], the condensability properties of the second one are very appealing when focusing material failure in large three-dimensional problems [34]. Therefore, in the remaining of this work, only finite elements with elemental enrichment (E-FEM) will be considered.

\section{Stability issues}

It is a very well known fact that finite element formulations for modeling material failure suffer, very often, from lack of robustness $[11,16]$. Even if powerful continuation methods to pass structural unstable points are used (i.e., arc length methods to traverse limit and turning points), it is noticed that, as the material failure progresses across the solid, the condition number of the structural tangent stiffness matrix deteriorates, the iterative Newton-Raphson procedure fails and, eventu- 
ally, the numerical simulation cannot be continued. In not few occasions, this type of difficulties has been attributed to illposedness of the BVP or lack of uniqueness of the corresponding finite element solution. However, even if the problem is mathematically well posed and the solution is unique, one eventually finds those types of difficulties; although some times they can be circumvented for very small (academic, two-dimensional) problems by using skilful procedures, they show up again as large problems, essentially in three dimensions, are tackled.

A first consideration should be made on the source of the problem: lack of uniqueness and global structural instability are not the only reasons for the classical lack of robustness of material failure simulations. Even if those problems are circumvented, an additional reason for lack of robustness in finite element simulations of material failure is the appearance of null eigenvalues in the algorithmic elemental stiffness matrices that propagate through the mesh deteriorating, after the assembling procedure, the condition number of the global algorithmic stiffness matrix.

A second consideration refers to the identification of two possible reasons for the appearance of those null elemental eigenvalues: the lack of symmetry of the finite element formulations, and the use of strain softening in the constitutive models. Although those reasons (and the possible remedies) can be extended to a wider variety of formulations for material failure, in Section 2.1 they will be examined in the context of the CSDA.

\subsection{A non-symmetric E-FEM formulation}

E-FEM formulations for solving the weak form of the problem (13) have been presented in detail elsewhere [29]. Here, let us focus our attention on the non-symmetric formulation whose finite dimensional space of the discretized displacements is described by

$\mathscr{V}^{h} \equiv\left\{\dot{\mathbf{u}}(\mathbf{x}, t), \dot{\mathbf{u}}=\sum_{i=1}^{n_{\text {node }}} N_{i}(\mathbf{x}) \dot{\mathbf{d}}_{i}(t)+\sum_{e=1}^{n_{\mathrm{elem}}} \mathscr{M}^{(e)}(\mathbf{x}) \dot{\boldsymbol{\beta}}_{e}(t)\right\}$
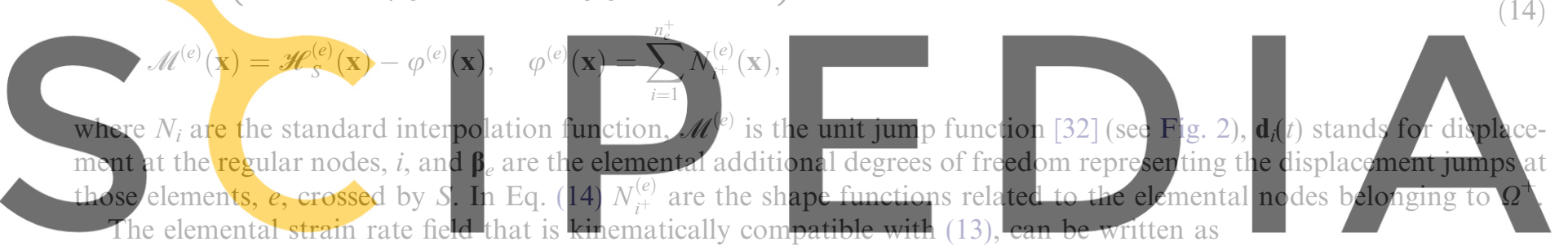

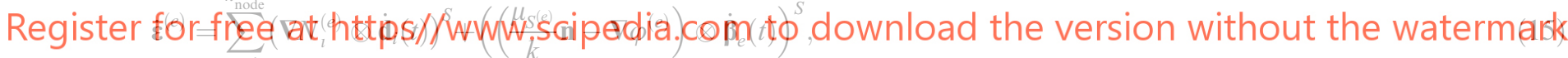

where $\mu_{S^{(e)}}(\mathbf{x})$ stands for a collocation function $\left(\mu_{S^{(e)}}=1\right.$ if $\mathbf{x} \in S^{(e)} ; \mu_{S^{(e)}}=0$ otherwise), $k$ is the regularization parameter considered in Eq. (3), and $\mathbf{n}$ is the normal vector to the elemental failure surface $S^{(e)}$, pointing to $\Omega^{+}$.

In the non-symmetric E-FEM approach, the field Eq. (13) are written in weak form via a Petrov-Galerkin formulation, which enforces the first Eqs. (13(a)-(d)) via a standard Galerkin procedure, whereas Eq. (13(e)) is locally enforced in strong form, at the center of every element. The resulting formulation can be then written as [29]:

$$
\begin{array}{ll}
\int_{\Omega / S} \nabla N_{i} \cdot \dot{\boldsymbol{\sigma}} \mathrm{d} \Omega=\dot{\mathbf{f}}_{i}^{\mathrm{ext}} & i \in\left\{1, \ldots, n_{\text {node }}\right\}, \\
S^{(e)}\left(\mathbf{n} \cdot \dot{\boldsymbol{\sigma}}_{S^{(e)}}-\mathbf{n} \cdot \frac{1}{\boldsymbol{\Omega}^{(e)}} \int_{\Omega^{(e)} / S^{(e)}} \dot{\boldsymbol{\sigma}}_{\Omega^{e} / S^{(e)}} \mathrm{d} \Omega\right)=\mathbf{0} & e \in\left\{1, \ldots, n_{\mathrm{elem}}\right\},
\end{array}
$$

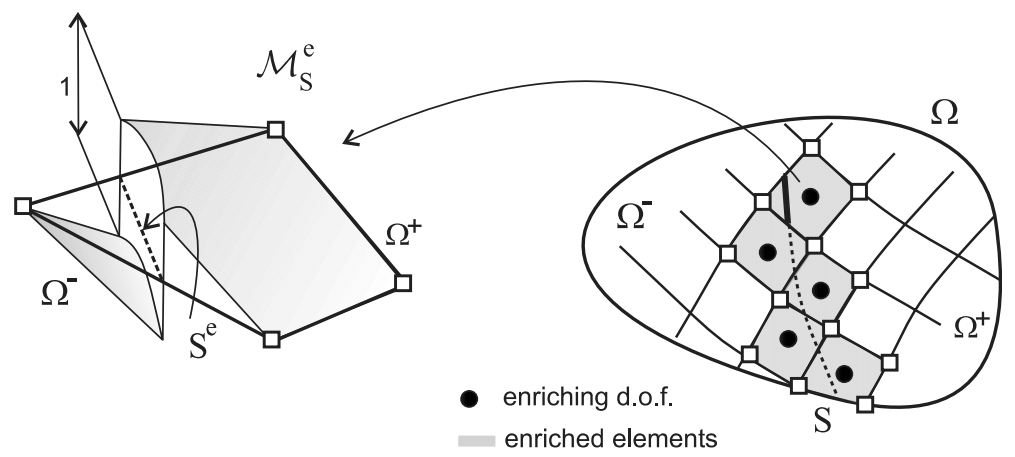

Fig. 2. Finite elements with elemental enrichment. 
where $S^{(e)}$ stands for a measure of the elemental counterpart of the failure interface. After the assembling process the problem in Eq. (16) can be written as (see [29,32] for more details):

$$
\begin{aligned}
& \mathbf{K} \cdot \dot{\mathbf{a}}=\dot{\mathbf{F}}, \dot{\mathbf{a}}=\left[\begin{array}{l}
\dot{\mathbf{u}} \\
\dot{\boldsymbol{\beta}}
\end{array}\right], \dot{\mathbf{F}}=\left[\begin{array}{c}
\dot{\mathbf{f}} \\
\mathbf{0}
\end{array}\right], \dot{\mathbf{u}}=\left\{\dot{\mathbf{u}}_{1}, \ldots, \dot{\mathbf{u}}_{n_{\text {node }}}\right\}^{\mathrm{T}}, \dot{\boldsymbol{\beta}}=\left\{\dot{\boldsymbol{\beta}}_{1}, \ldots, \dot{\boldsymbol{\beta}}_{n_{\text {elem }}}\right\}^{\mathrm{T}}, \\
& \mathbf{K}=\mathscr{A}\left(\mathbf{K}^{(e)}\right), \mathbf{K}^{(e)}=\left[\begin{array}{ll}
\mathbf{K}_{u u}^{(e)} & \mathbf{K}_{u \beta}^{(e)} \\
\mathbf{K}_{\beta u}^{(e)} & \mathbf{K}_{\beta \beta}^{(e)}
\end{array}\right]=\int_{\Omega^{(e)}} \mathbf{B}^{(e)^{*}} \cdot \mathbf{C}^{\mathrm{alg}} \cdot \mathbf{B}^{(e)} \mathrm{d} \Omega, \\
& \mathbf{B}^{(e)}=\left[\nabla N_{1}^{(e)}, \nabla N_{2}^{(e)}, \ldots, \nabla N_{n_{\text {node }}^{(e)}}^{(e)},\left(\frac{\mu_{S^{(e)}}}{k}-\nabla \varphi^{(e)}\right)\right], \\
& \mathbf{B}^{(e)^{*}}=\left[\nabla N_{1}^{(e)}, \nabla N_{2}^{(e)}, \ldots, \nabla N_{n_{\text {node }}^{(e)}}^{(e)},\left(\frac{\mu_{S^{(e)}}}{k}-\frac{S^{(e)}}{\Omega^{e}}\right) \mathbf{n}\right],
\end{aligned}
$$

where $\mathscr{A}(\bullet)$ stands for the assembling operator. The specific sub-matrices of the elemental tangent stiffness matrix $\mathbf{K}^{(e)}$ are

$$
\begin{aligned}
& {\left[\mathbb{K}_{u u u_{i j}}^{e}=\int_{\Omega^{(e)} / S^{(e)}} \nabla N_{i}^{(e)} \cdot \mathbf{C}_{\Omega / S}^{\mathrm{alg}} \cdot \nabla N_{j}^{(e)} \mathrm{d} \Omega,\right.} \\
& {\left[\mathbb{K}_{u \beta}^{e}\right]_{i}=-\int_{\Omega^{(e) / S^{(e)}}} \nabla N_{i}^{(e)} \cdot \mathbf{C}_{\Omega / S}^{\mathrm{alg}} \cdot \nabla \varphi^{(e)} \mathrm{d} \Omega,} \\
& {\left[\mathbb{K}_{\beta u}^{e}\right]_{j}=-\frac{S^{(e)}}{\Omega^{e}} \int_{\Omega^{(e)} / S^{(e)}} \mathrm{n} \cdot \mathbf{C}_{\Omega / S}^{\mathrm{alg}} \cdot \nabla N_{j}^{(e)} \mathrm{d} \Omega,}
\end{aligned}
$$
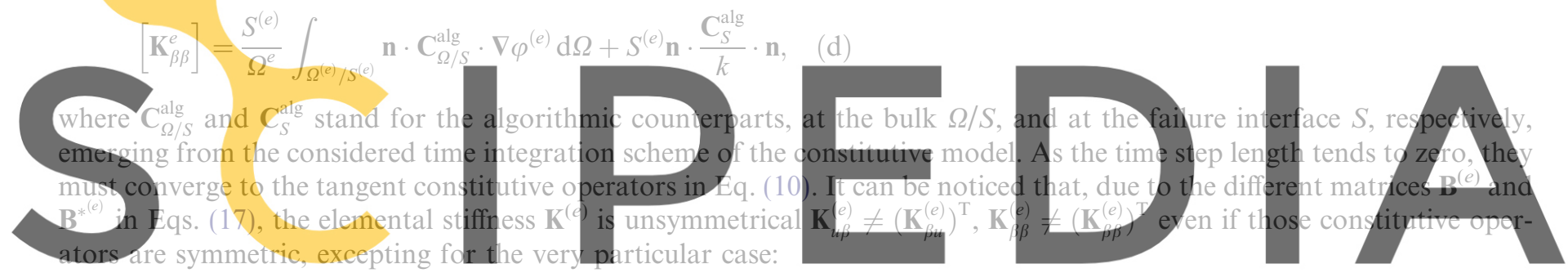

Registerfor frese at https//www.scipedia.com to download the version without the watermark

For linear elements, this corresponds to the case of the elemental failure line/surface being parallel to one side/surface of the element $(\alpha=0$ in Fig. 3$)$.

\subsection{Considerations about stability}

On the light of the expressions of the elemental stiffness matrices in Eq. (18), one can identify two different sources of null/negative eigenvalues in the elemental tangent stiffness $\mathbf{K}^{(e)}$ :

(1) The unsymmetrical character of the elemental stiffness matrix $\mathbf{K}^{(e)}$ and, therefore, of the global stiffness matrix $\mathbf{K}$. Even if the constitutive operators $\mathbf{C}_{\Omega / S}^{\mathrm{alg}}$ and $\mathbf{C}_{S}^{\mathrm{alg}}$ are symmetric and positive definite (as it happens in Eq. (10) for the elastic or unloading cases), a reason for the eventual production of null/negative eigenvalues still remains: the non-symmetric character of the term $\mathbf{n} \cdot \mathbf{C}_{\Omega / S}^{\mathrm{alg}} \cdot \nabla \varphi^{(e)}$ in Eq. (18(d)). Unless the vectors $\mathbf{n}$ and $\nabla \varphi^{(e)}$ are parallel (as

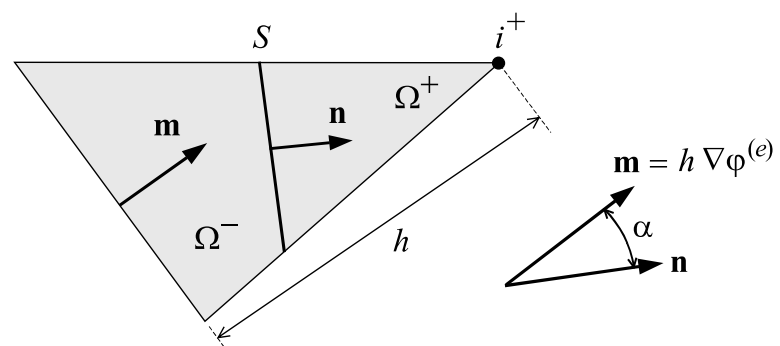

Fig. 3. Orientation of the failure interface in an enriched element. 
it occurs in the very particular symmetric case presented in Eq. (19)), that term may contribute to produce null or negative eigenvalues as the angle between those vectors is large enough (see Fig. 3). This problem has been mentioned some times in the literature as responsible for lack of uniqueness of the finite element solution [16] or for a bad tracing of the failure interface [35], but here it is analyzed from the stability and robustness point of view. At any case, it is clearly typical of unsymmetrical finite element formulations, as the one presented in Section 2.1, and symmetric finite element formulations would preclude this source of numerical instability.

(2) The strain softening in the constitutive model. Even if the finite element formulation is symmetric, the strain softening in the constitutive model may be responsible for the appearance of negative eigenvalues at any of the constitutive operators $\mathbf{C}_{\Omega / S}^{\mathrm{alg}}$ or $\mathbf{C}_{S}^{\mathrm{alg}}$ in Eq. (18) and becomes a possible source of null eigenvalues in the elemental stiffness matrix. Neither imposition of an elastic behavior in the bulk, as it is done in many discrete formulations of the SDA based on discrete traction-separation laws (equipped with displacement softening) at the failure interface, solves the problem. This would be equivalent to equate $\mathbf{C}_{\Omega / S}^{\mathrm{alg}}$ in Eq. (18) to the, positive definite, elastic constitutive operator $\mathbf{C}^{e}$ in Eq. (10), but the term $\mathbf{n} \cdot \frac{\mathbf{C}_{s}^{\mathrm{alg}}}{k} \cdot \mathbf{n}$, in Eq. (18(d)), exhibiting negative eigenvalues for loading processes due to the negative value of the softening modulus $H\left(\mathbf{C}_{S}^{\mathrm{alg}}=\mathbf{C}^{l}\right.$, in Eq. (10)), still remains. On the light of the previous reasoning we conclude that this source of numerical instability would be removed if the positive definite character of the algorithmic constitutive operators $\mathbf{C}_{\Omega / S}^{\mathrm{alg}}$ and $\mathbf{C}_{S}^{\mathrm{alg}}$ was ensured at any point of either the bulk, $\Omega / S$, or the faiture interface $S$.

In summary, from the previous analysis it can be concluded that a symmetric E-FEM formulation, combined with an integration procedure that renders the algorithmic tangent constitutive operator positive definite, would remove the identified sources of lack of robustness of the CSDA. Actions to achieve these goals are described in Section 2.3.

2.3. Symmetric (kinematically consistent) formulation

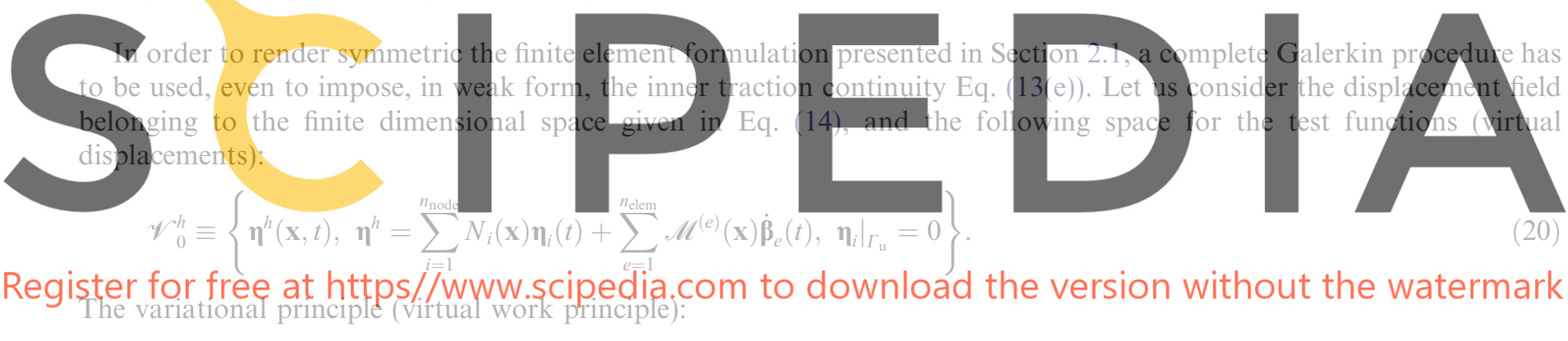

$$
\delta \Pi\left(\dot{\mathbf{u}} ; \boldsymbol{\eta}^{h}\right) \equiv \int_{\Omega} \nabla \boldsymbol{\eta}^{h}: \dot{\boldsymbol{\sigma}} \mathrm{d} \Omega-\left[\int_{\Omega} \boldsymbol{\eta}^{h} \cdot \boldsymbol{\rho}_{0} \dot{\mathbf{b}} \mathrm{d} V+\int_{\Gamma_{\sigma}} \boldsymbol{\eta}^{h} \cdot i^{*} \mathrm{~d} \Gamma\right]=\mathbf{0} \quad \forall \boldsymbol{\eta}^{h} \in \mathscr{r}_{0}^{h}
$$

defines a formulation of a finite element with an embedded discontinuity that: (a) belongs to the symmetric kinematically consistent class [16,32], and (b) is the weak form of the problem (13). The matrix form of the BVP resulting from this type of formulation is similar, but different, to the one in Eq. (16):

$$
\begin{array}{ll}
\int_{\Omega / S} \nabla N_{i} \cdot \dot{\boldsymbol{\sigma}} \mathrm{d} \Omega=\dot{\mathbf{f}}_{i}^{\mathrm{xt}} & i \in\left\{1, \ldots, n_{\text {node }}\right\} \\
\int_{\Omega / S^{(e)}} \nabla \varphi^{(e)} \cdot \dot{\boldsymbol{\sigma}}_{\Omega^{e} / S^{(e)}} \mathrm{d} \Omega-\int_{S^{(e)}} \mathbf{n} \cdot \dot{\boldsymbol{\sigma}}_{S} \mathrm{~d} S=\mathbf{0} & e \in\left\{1, \ldots, n_{\mathrm{elem}}\right\}
\end{array}
$$

where $S^{(e)}$ is the elemental crack length, which can be either geometrically computed or approximated as $S^{(e)}=\Omega^{(e)} \mathbf{n} \cdot \nabla \varphi^{(e)}$ (see [21]). Now, the matrix form of the problem reads:

$$
\begin{aligned}
& \mathbf{K} \cdot \dot{\mathbf{a}}=\dot{\mathbf{F}}, \dot{\mathbf{a}}=\left[\begin{array}{c}
\dot{\mathbf{u}} \\
\dot{\boldsymbol{\beta}}
\end{array}\right], \dot{\mathbf{F}}=\left[\begin{array}{c}
\dot{\mathbf{f}} \\
\mathbf{0}
\end{array}\right], \dot{\mathbf{u}}=\left\{\dot{\mathbf{u}}_{1}, \ldots, \dot{\mathbf{u}}_{n_{\mathrm{node}}}\right\}^{\mathrm{T}}, \dot{\boldsymbol{\beta}}=\left\{\dot{\boldsymbol{\beta}}_{1}, \ldots, \dot{\boldsymbol{\beta}}_{n_{\mathrm{elem}}}\right\}^{\mathrm{T}}, \\
& \mathbf{K}=\mathscr{A}\left(\mathbf{K}^{(e)}\right), \quad \mathbf{K}^{(e)}=\left[\begin{array}{cc}
\mathbf{K}_{u u}^{(e)} & \mathbf{K}_{u \beta}^{(e)} \\
\mathbf{K}_{\beta u}^{(e)} & \mathbf{K}_{\beta \beta}^{(e)}
\end{array}\right]=\int_{\Omega^{(e)}} \mathbf{B}^{(e)^{\mathrm{T}}} \cdot \mathbf{C}^{\mathrm{alg}} \cdot \mathbf{B}^{(e)} \mathrm{d} \Omega, \\
& \mathbf{B}^{(e)}=\left[\nabla N_{1}^{(e)}, \nabla N_{2}^{(e)}, \ldots, \nabla N_{n_{\text {node }}^{(e)}}^{(e)},\left(\frac{\mu_{S^{(e)}}}{k} \mathbf{n}-\nabla \varphi^{(e)}\right)\right]
\end{aligned}
$$


and the specific sub-matrices expressions are

$$
\begin{aligned}
& {\left[\mathbf{K}_{u u}^{e}\right]_{i j}=\int_{\Omega^{(e)} / S^{(e)}} \nabla N_{i}^{(e)} \cdot \mathbf{C}_{\Omega / S}^{\mathrm{alg}} \cdot \nabla N j_{i}^{(e)} \mathrm{d} \Omega,} \\
& {\left[\mathbf{K}_{u \beta}^{e}\right]_{i}=-\int_{\Omega^{(e) / S^{(e)}}} \nabla N_{i}^{(e)} \cdot \mathbf{C}_{\Omega / S}^{\mathrm{alg}} \cdot \nabla \varphi^{(e)} \mathrm{d} \Omega,} \\
& {\left[\mathbf{K}_{\beta u}^{e}\right]_{j}=-\int_{\Omega^{(e)} / S^{(e)}} \nabla \varphi^{(e)} \cdot \mathbf{C}_{\Omega / S}^{\mathrm{alg}} \cdot \nabla N_{i}^{(e)} \mathrm{d} \Omega,} \\
& {\left[\mathbf{K}_{\beta \beta}^{e}\right]=\int_{\Omega^{(e)} / S^{(e)}} \nabla \varphi^{(e)} \cdot \mathbf{C}_{\Omega / S}^{\mathrm{alg}} \cdot \nabla \varphi^{(e)} \mathrm{d} \Omega+\int_{S^{(e)}} \mathbf{n} \cdot \frac{\mathbf{C}_{S}^{\mathrm{alg}}}{k} \cdot \mathbf{n} \mathrm{d} S,}
\end{aligned}
$$

Notice that, due to the variational procedure for the derivation, the elemental stiffness matrix, $\mathbf{K}^{(e)}$, and, therefore, the global stiffness matrix, $\mathbf{K}$, is symmetric and positive definite if so are the algorithmic constitutive operators $\mathbf{C}_{\Omega / S}^{\text {alg }}$ and $\mathbf{C}_{S}^{\text {alg }}$. This is a crucial point to be exploited in Section 2.4.3.

In a subsequent manipulation, the rate equations (22) can be written in an alternative total form as

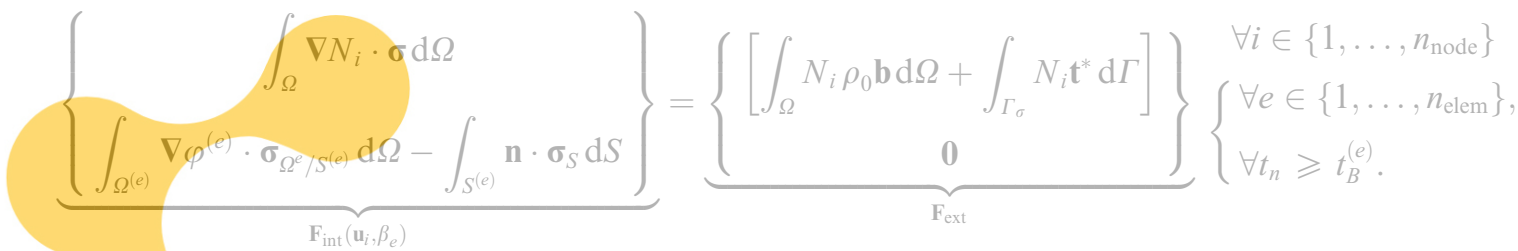

where $t_{B}^{(e)}$ stands for the time of the onset of the material failure at element $e$.

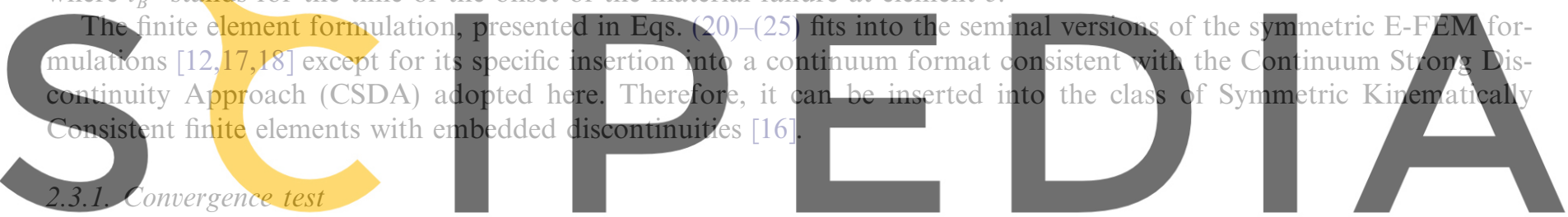

Unlike the non-symmetrical formulation in Section 2.1 the symmetrical weak formulation does not enforce the inner traction continuity Eq.(13(e)) in strong form. However, the equivalence of the weak form (21) with the totality of the field

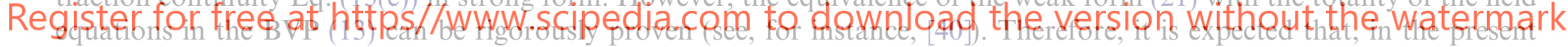

symmetric formulation, mesh refinement will determine a correct trend (convergence) to the fulfillment of those equations.

The test in Fig. 4 constitutes a simple corroboration of this fact, taking the linear triangle as the underlying element, and provides an assessment of the order of convergence of that symmetric finite element with embedded discontinuities.

The test consists of a homogeneous rectangular strip pulled from the right end with a force $\mathbf{P}$, imposing a displacement $\Delta$, up to the formation of an inclined failure line and, then, continued to the total failure and release of the stresses. Due to the induced constant stress field, the considered bilinear stress-strain law for the constitutive model should translate into a bilinear force-displacement $(\mathbf{P}-\boldsymbol{\Delta})$ curve. To check the convergence to the right slope of the descending branch and, therefore, to the right energy dissipation $G_{f}$, a homogeneous mesh refinement, parameterized in terms of the element size $h$, is
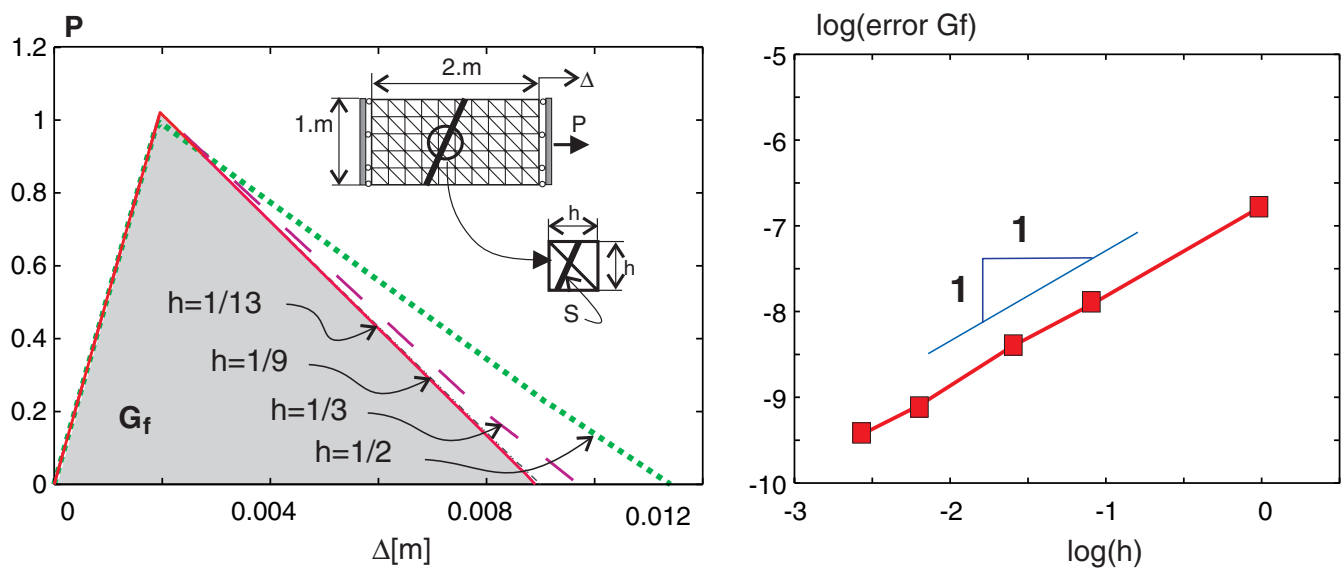

Fig. 4. Symmetric finite element formulation: convergence test. 
performed. Fig. 4(left) shows the results, for increasingly fine meshes, converging to the exact solution (the curve limiting the gray zone). The errors in the dissipated energy (fracture energy) for the different levels of discretization are displayed in Fig. 4(right), where the linear convergence of the element is observed.

It is worth mentioning that this symmetric element, although convergent, exhibits an accuracy smaller than its unsymmetrical counterpart, according to the formulation in Section 2.1, for this type of homogeneous/constant stress problem. Indeed, it can be proven that, for this particular case, the unsymmetrical element provides the exact solution with only one element.

\subsection{Implicit-explicit integration scheme for the constitutive model}

\subsubsection{Consistent algorithmic operator of the implicit integration procedure}

Let us first focus on the damage constitutive model in the left Eqs. (4)-(10). Implicit integration of the rate Eqs. (5) and (9) leads to the following solutions, at time $t_{n+1}$, for the internal variables $r_{n+1}, q_{n+1}$ and the stress $\boldsymbol{\sigma}_{n+1}$ in terms of the current strains $\boldsymbol{\varepsilon}_{n+1}$ (see [25]):

$$
\begin{aligned}
& \varepsilon_{n+1} \mapsto\left\{\begin{array}{l}
\dot{r}=\dot{\lambda} \geqslant 0,\left.\quad r\right|_{t=0}=r_{0} \\
g\left(\varepsilon_{n+1}, r_{n+1}\right) \equiv \tau_{\varepsilon}\left(\varepsilon_{n+1}\right)-r_{n+1} \leqslant 0
\end{array} \rightarrow r_{n+1}\left(\varepsilon_{n+1}\right)=\max _{s \in\left[0, t_{n}\right]}\left(r_{s}, \tau_{\varepsilon}\left(\varepsilon_{n+1}\right)\right)\right. \\
& \Delta \lambda_{n+1}\left(\boldsymbol{\varepsilon}_{n+1}\right)=\Delta r_{n+1}=r_{n+1}\left(\boldsymbol{\varepsilon}_{n+1}\right)-r_{n} \\
& \begin{array}{c}
q_{n+1}\left(\boldsymbol{\varepsilon}_{n+1}\right)=q_{n}+H \Delta \\
\boldsymbol{\sigma}_{n+1}\left(\varepsilon_{n+1}\right)=\frac{q_{n+1}\left(\boldsymbol{\varepsilon}_{n+1}\right)}{r_{n+1}\left(\boldsymbol{\varepsilon}_{n+1}\right)} \underbrace{\mathbf{C}^{e}: \boldsymbol{\varepsilon}_{n+1}}_{\overline{\boldsymbol{\sigma}}_{n+1}} .
\end{array}
\end{aligned}
$$

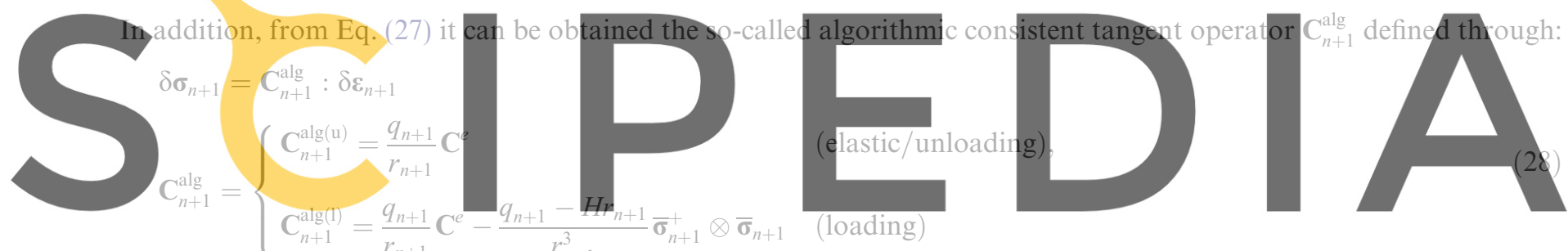

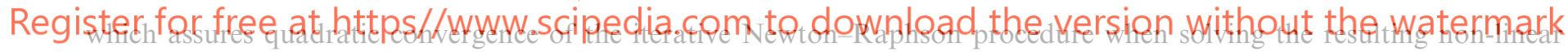
problem. A similar, slightly more complex, procedure can be performed for the implicit integration of the plasticity model in the right Eqs. (4)-(10). After some algebraic operations one gets [4.2]:

$$
\boldsymbol{\varepsilon}_{n+1} \mapsto\left\{\begin{array}{l}
\dot{r}=\dot{\lambda} \geqslant 0,\left.\quad r\right|_{t=0}=0 \\
\Delta \lambda_{n+1}=\Delta r_{n+1}=r_{n+1}-r_{n}
\end{array} \rightarrow q_{n+1}\left(\Delta \lambda_{n+1}\right)=q_{n}-H\left(\Delta r_{n+1}\right)=q_{n}+H \Delta \lambda_{n+1} .\right.
$$

The algorithmic plastic multiplier, $\Delta \lambda_{n+1}$, can be solved by imposing, for loading cases, the yield criterion (7) (consistency) at time $t_{n+1}$ :

$$
\begin{aligned}
\boldsymbol{\varepsilon}_{n+1} \mapsto & \begin{array}{l}
\boldsymbol{\sigma}_{n+1}\left(\Delta \lambda_{n+1}\right)=\boldsymbol{\sigma}_{n}+\mathbf{C}^{e}:\left(\boldsymbol{\varepsilon}_{n+1}-\boldsymbol{\varepsilon}_{n}\right)-\Delta \lambda_{n+1} \mathbf{C}^{e}: \boldsymbol{\xi}_{n+1}\left(\boldsymbol{\sigma}_{n+1}\left(\Delta \lambda_{n+1}\right)\right) \\
\xi_{n+1}=\frac{\partial g\left(\boldsymbol{\sigma}_{n+1}, q_{n+1}\right)}{\partial \boldsymbol{\sigma}_{n+1}}, \quad \mathbf{A}_{n+1}=\frac{\partial^{2} g\left(\boldsymbol{\sigma}_{n+1}, q_{n+1}\right)}{\partial \boldsymbol{\sigma}_{n+1} \otimes \partial \boldsymbol{\sigma}_{n+1}}
\end{array} \\
& \rightarrow g\left(\boldsymbol{\sigma}_{n+1}, q_{n+1}\right) \equiv g\left(\boldsymbol{\sigma}_{n+1}\left(\boldsymbol{\varepsilon}\left(\Delta \lambda_{n+1}\right), q_{n+1}\left(\Delta \lambda_{n+1}\right)\right)\right)=0 \rightarrow \Delta \lambda_{n+1}\left(\boldsymbol{\varepsilon}_{n+1}\right) \rightarrow \\
& \rightarrow \boldsymbol{\sigma}_{n+1}\left(\Delta \lambda_{n+1}\left(\boldsymbol{\varepsilon}_{n+1}\right)\right) .
\end{aligned}
$$

Some additional manipulations lead to the algorithmic tangent operator $\mathbf{C}_{n+1}^{\text {alg }}$ :

$$
\begin{aligned}
& \delta \boldsymbol{\sigma}_{n+1}=\mathbf{C}_{n+1}^{\mathrm{alg}}: \delta \boldsymbol{\varepsilon}_{n+1}, \\
& \mathbf{C}_{n+1}^{\mathrm{alg}}= \begin{cases}\mathbf{C}_{n+1}^{\mathrm{alg}(\mathrm{u})}=\mathbf{C}^{e} & \text { (elastic/unloading), } \\
\mathbf{C}_{n+1}^{\mathrm{alg}(1)}=\widehat{\mathbf{C}}-\frac{\widehat{\mathbf{C}}: \xi_{n+1} \otimes \widehat{\mathbf{C}}: \boldsymbol{\xi}_{n+1}}{H+\xi_{n+1}: \widehat{\mathbf{C}}: \boldsymbol{\xi}_{n+1}} & \text { (loading), } \\
\widehat{\mathbf{C}}=\left(\mathbf{I}+\Delta \lambda_{n+1} \mathbf{C}^{e}: \mathbf{A}_{n+1}\right)^{-1}: \mathbf{C}^{e} .\end{cases}
\end{aligned}
$$


Inspection of the algorithmic constitutive operators $\mathbf{C}_{n+1}^{\mathrm{alg}}$ in (28) and (31) reveals that $\mathbf{C}_{n+1}^{\mathrm{alg}(\mathrm{u})}$ is always positive definite, but $\mathbf{C}_{n+1}^{\mathrm{alg}(1)}$ may lose the positive character as strain softening $(H<0)$ is considered. Indeed, this is the case that one faces in computational material failure: as it will be shown in Section 2.5, loss of strong ellipticity of the algorithm tangent operator $\mathbf{C}^{\text {alg }}$ at increasing parts of the analyzed domain $\Omega$, deteriorates the positive character of the structural tangent stiffness $\mathbf{K}$ of the discrete problem (21), even using the symmetric formulation, which, eventually, becomes singular. This translates into enormous problems of convergence and robustness in the resulting solving process.

The situation can be summarized as follows: the use of classical implicit integration algorithms, for constitutive models equipped with strain softening, leads to accurate results even for large time steps. However, it also results into ill conditioning of the stiffness matrix of the resulting problem which, in turn, enforces small time steps and computationally costly procedures to get convergence (very often, even no convergence is obtained). In view of this observation, the question of the worthiness to renounce to some of the accuracy of the purely implicit integration procedures to benefit the robustness of the solving procedure arises. This motivates the implicit-explicit integration procedure presented in next section.

\subsubsection{Implicit-explicit integration: effective algorithmic operator}

The procedure consists of using two integration schemes per time step: one is the standard implicit (Backward-Euler) sketched in Eqs. (26)-(31) furnishing "implicit" values for the variables of the problem, $r\left(\varepsilon_{n+1}\right), q\left(\varepsilon_{n+1}\right), \sigma_{n+1}\left(\varepsilon_{n+1}\right)$, in terms of the current strains $\varepsilon_{n+1}$. A second explicit integration is performed at the same time step, furnishing other "explicit" values $\tilde{r}\left(\varepsilon_{n+1}\right), \tilde{q}\left(\varepsilon_{n+1}\right), \tilde{\boldsymbol{\sigma}}_{n+1}\left(\boldsymbol{\varepsilon}_{n+1}\right)$ for the variables of the model. The variational problem (21) is then solved using the explicit stresses $\tilde{\boldsymbol{\sigma}}_{n+1}$, and the algorithmic constitutive operator, consistent with the integration scheme in the variational problem, is termed the effective algorithmic operator, $\mathbf{C}_{n+1}^{\text {eff }}=\partial_{\varepsilon_{n+1}} \tilde{\boldsymbol{\sigma}}_{n+1}\left(\boldsymbol{\varepsilon}_{n+1}\right)$ (to be distinguished from the one in the implicit integration procedure $\mathbf{C}_{n+1}^{\text {atg }}=\partial_{\varepsilon_{n+1}} \boldsymbol{\sigma}_{n+1}\left(\boldsymbol{\varepsilon}_{n+1}\right)$ ). Some interesting properties of the effective operator are displayed next for the continuum damage and the elasto-plastic models.

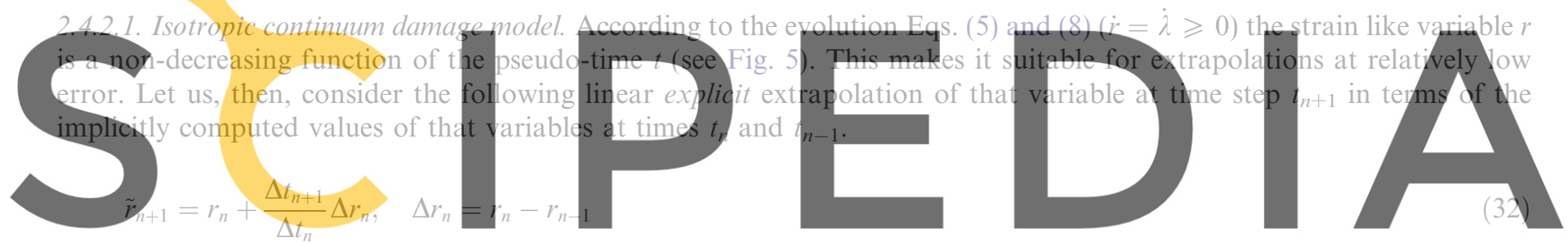

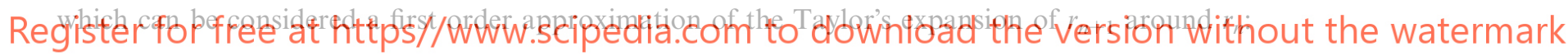

$$
r_{n+1}=r_{n}+\underbrace{\dot{r}_{n}}_{\frac{\Delta r_{n}}{\Delta t_{n}}} \Delta t_{n+1}+\mathcal{O}\left(\Delta t_{n+1}^{2}\right)=\tilde{r}_{n+1}+\mathcal{O}\left(\Delta t_{n+1}^{2}\right) .
$$

Two considerations emerge from Eqs. (32) and (33):

- $\tilde{r}_{n+1}$ constitutes a prediction for the value of the internal value at time step $n+1$ that can be computed at the end of the time step $n$ and, thus, is independent of the value of the current strains $\varepsilon_{n+1}$.

- Indeed, there is an additional error introduced by computing the stresses at time step $n+1$ in terms of the value $\tilde{r}_{n+1}$ instead of the implicit value $r_{n+1}$. However, this error can be reduced (or controlled) either by decreasing the time step length or by increasing the order of the extrapolation procedure.
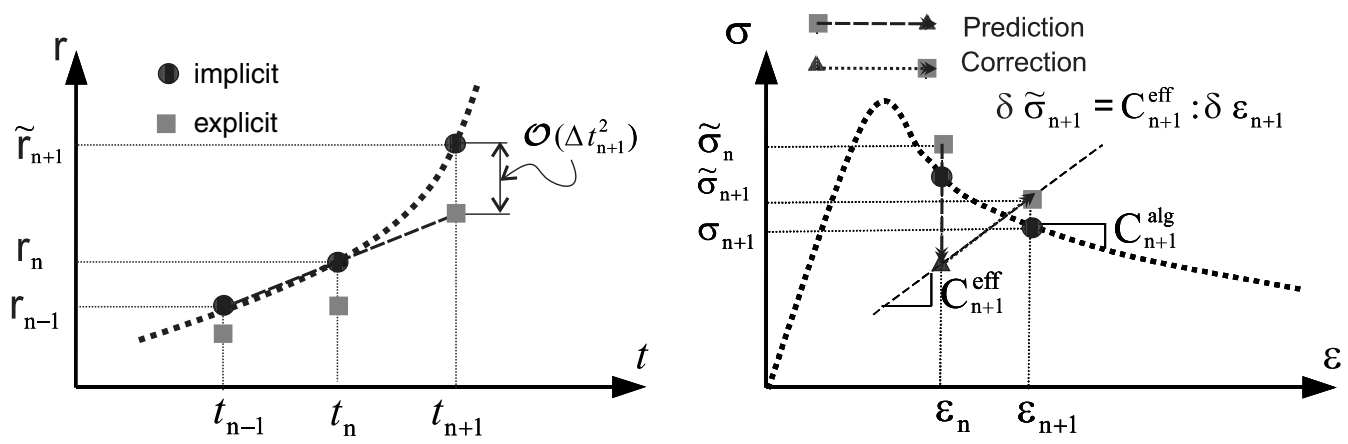

Fig. 5. Implicit/explicit integration algorithm. (Left) Extrapolation of the strain-like internal variable. (Right) Prediction/correction phases of the implicit-explicit integration procedure. 
Once $\tilde{r}_{n+1}$ is known, from Eq. (32), the stresses at the current time $t_{n+1}$ can be computed using Eqs. (6) and (9) as

$$
\begin{aligned}
& \left\{\begin{array}{l}
\tilde{q}_{n+1}=q_{n}+H_{n}\left(\tilde{r}_{n+1}-r_{n}\right)=q_{n}+H_{n} \Delta r_{n}=q_{n}+H_{n}\left(r_{n}-r_{n-1}\right), \\
\tilde{q}_{n+1} \geqslant 0,
\end{array}\right. \\
& \boldsymbol{\varepsilon}_{n+1} \mapsto \tilde{\boldsymbol{\sigma}}_{n+1}=\frac{\tilde{q}_{n+1}}{\tilde{r}_{n+1}} \mathbf{C}^{e}: \boldsymbol{\varepsilon}_{n+1},
\end{aligned}
$$

where it should be noticed that neither $\tilde{q}_{n+1}$ nor $\tilde{r}_{n+1}$ depend on the current strains $\boldsymbol{\varepsilon}_{n+1}$ and, therefore, the algorithmic stresses $\tilde{\boldsymbol{\sigma}}_{n+1}$ only depend, linearly, on the strains $\boldsymbol{\varepsilon}_{n+1}$. Thus, the algorithmic tangent operator emerging from the above implicit-explicit integration procedure (from now on termed the effective algorithmic operator) reads:

$$
\mathbf{C}_{n+1}^{\text {eff }}=\frac{\partial \tilde{\boldsymbol{\sigma}}_{n+1}}{\partial \boldsymbol{\varepsilon}_{n+1}}=\frac{\tilde{q}_{n+1}}{\tilde{r}_{n+1}} \mathbf{C}^{e}, \quad \delta \tilde{\boldsymbol{\sigma}}_{n+1}=\mathbf{C}_{n+1}^{\text {eff }}: \partial \boldsymbol{\varepsilon}_{n+1}
$$

which is constant during the time step $n+1$. In addition, due to the positive character of $\tilde{q}_{n+1}, \tilde{r}_{n+1}$ and $\mathbf{C}^{e}$, one can conclude that $\mathbf{C}_{n+1}^{\text {eff }}$ is always positive definite.

2.4.2.2. Elasto-plastic material model. Similarly, for the elasto-plastic rate independent model in Eqs. (4)-(10), extrapolation of the strain-like internal variable $r_{n+1}$ reads:

$$
\left\{\begin{array}{l}
\tilde{r}_{n+1}=r_{n}+\underbrace{\dot{r}_{n}}_{\frac{\Delta r_{n}}{\Delta t_{n}}} \Delta t_{n+1}=r_{n}+\frac{\Delta t_{n+1}}{\Delta t_{n}} \underbrace{\left(r_{n}-r_{n-1}\right)}_{\Delta \lambda_{n} \geqslant 0}=r_{n}+\frac{\Delta t_{n+1}}{\Delta t_{n}} \Delta \lambda_{n}, \\
\Delta \tilde{\lambda}_{n+1} \stackrel{\text { def }}{\equiv} \tilde{r}_{n+1}-r_{n}=\frac{\Delta t_{n+1}}{\Delta t_{n}} \Delta \lambda_{n} \geqslant 0,
\end{array}\right.
$$

where $\Delta \lambda_{n}$ is the implicitly integrated value of the plastic multiplier at time step $n$. Then, from Eq. (9):

$$
\left\{\begin{array}{l}
\tilde{q}_{n+1}=q_{n}+H_{n}\left(\tilde{r}_{n+1}-r_{n}\right)=q_{n}+H_{n} \Delta r_{n}=q_{n}+H_{n}\left(r_{n}-r_{n-1}\right), \\
\boldsymbol{\sigma}_{\mathrm{u}} \geqslant \tilde{q}_{n+1} \geqslant 0 .
\end{array}\right.
$$

Once the predictions $\Delta \tilde{\lambda}_{n+1}$ and $\tilde{q}_{n+1}$ are available, the stress field can be computed from Eq. (30) as

$$
\boldsymbol{\varepsilon}_{n+1} \mapsto \mid \begin{aligned}
& \tilde{\boldsymbol{\sigma}}_{n+1}=\boldsymbol{\sigma}_{n}+\mathbf{C}^{e}:\left(\boldsymbol{\varepsilon}_{n+1}-\boldsymbol{\varepsilon}_{n}\right)-\Delta \tilde{\lambda}_{n+1} \mathbf{C}^{e}: \tilde{\xi}\left(\tilde{\boldsymbol{\sigma}}_{n+1}\right), \\
& \tilde{\boldsymbol{\xi}}_{n+1}=\frac{\partial g\left(\tilde{\boldsymbol{\sigma}}_{n+1}, \tilde{q}_{n+1}\right)}{\partial \tilde{\boldsymbol{\sigma}}_{n+1}}, \quad \tilde{\mathbf{A}}_{n+1}=\frac{\partial \tilde{\xi}_{n+1}}{\partial \tilde{\boldsymbol{\sigma}}_{n+1}}=\frac{\partial^{2} g\left(\tilde{\boldsymbol{\sigma}}_{n+1}, \tilde{q}_{n+1}\right)}{\partial \tilde{\boldsymbol{\sigma}}_{n+1} \otimes \partial \tilde{\boldsymbol{\sigma}}_{n+1}},
\end{aligned}
$$

where $\boldsymbol{\sigma}_{n}$ is the implicit stress at time step $n$. The corresponding effective algorithmic tensor reads:

$$
\mathbf{C}_{n+1}^{\text {eff }}=\frac{\partial \tilde{\boldsymbol{\sigma}}_{n+1}}{\partial \boldsymbol{\varepsilon}_{n+1}}=\left(\mathbf{I}+\Delta \tilde{\lambda}_{n+1} \tilde{\mathbf{A}}_{n+1}\right)^{-1}: \mathbf{C}^{e}, \quad \delta \tilde{\boldsymbol{\sigma}}_{n+1}=\mathbf{C}_{n+1}^{\mathrm{eff}}: \partial \boldsymbol{\varepsilon}_{n+1} .
$$

It should be noticed that, for the usual plasticity models, the tensor $\widetilde{\mathbf{A}}_{n+1}$ in Eqs. (30) and (39) is positive definite as part of the requirements for convexity of the yield (or the potential) surface $g(\boldsymbol{\sigma}, q)$ in Eq. (7), in the generalized stress $\left(\boldsymbol{\Sigma} \equiv\{\boldsymbol{\sigma}, q\}^{\mathrm{T}}\right)$ space [42]. In addition, as a requirement for the dimensional consistency of $g(\boldsymbol{\Sigma})$, the yield function must be an homogeneous, of a certain order, function of $\boldsymbol{\Sigma}$ which, with a simple algebraic treatment, can be appropriately reduced to one of second order. In summary: $\widetilde{\mathbf{A}}_{n+1}$ in Eq. (40) can be always made constant and positive definite and, therefore, so is the effective algorithmic tensor $\mathbf{C}_{n+1}^{\text {eff }}$.

\subsubsection{Remarks on the implicitlexplicit integration scheme}

The following aspects should be noticed about the proposed implicit/explicit integration scheme in Eqs. (32)-(40):

1. Since the extrapolated values, $\tilde{r}_{n+1}$, are obtained in terms of the implicitly integrated values, $r_{n}$ and $r_{n-1}$, one can expect that the well-known stability properties of the implicit integration procedures are being inherited by their explicit extrapolations (32) and (37). In other words, the integration errors will not amplify even for relative large time steps, unlike it typically happens for purely explicit schemes.

2. The ability of the constitutive model to reproduce material instability and failure, via the loss of the positive character of the tangent constitutive operator, still holds. The implicit/explicit integration procedure is, in fact, an algorithmically stable (robust) integration procedure to approach the unstable response of the material and of the constitutive model. This fact is emphasized in Fig. 5(right) where the implicit/explicit integration of the constitutive model can be understood as composed of a prediction stage, at the end of time step $n$, followed by a linear correction, characterized by the constant and positive definite operator $\mathbf{C}_{n+1}^{\text {eff }}$, during time step $n+1$. 
3. The algorithmic tangent constitutive operator, $\mathbf{C}_{n+1}^{\text {alg }}$ in Eqs. (28) and (31), consistent with the implicit integration scheme, can be still calculated at every time step and its spectral properties used to determine the onset of local material failure and the directions of propagation of material failure.

4. The constant and positive definite character of the effective algorithmic operator $\mathbf{C}_{n+1}^{\text {eff }}$ in Eqs. (36) and (40), will now have the following effects on the iterative procedure for solving the non-linear problem using the symmetric E-FEM formulation in Eqs. (20)-(25):

- The algorithmic (effective) stiffness matrix of the problem will always be positive definite (see Appendix A). No numerical instabilities should be expected at any time and the robustness of the analysis should be dramatically increased.

- Since the structural tangent stiffness matrix is now constant and well conditioned, the Newton-Raphson procedure should converge in just one iteration per time step.

5. In compensation, smaller accuracies for relatively large time steps, in comparison with the ones obtained with the purely implicit integration procedure, should be expected. Consequently, the time advancing procedure should be combined with a procedure for controlling the integration errors in Eqs. (33) and (37).

\subsection{Benchmark problem: double cantilever beam (DCB) test}

The following example clearly illustrates the lack of stability and robustness problems that motivate this work and the benefits of the proposed remedies. It consists of the classical DCB test: a concrete specimen with an idealized (very low) fracture energy $G_{\mathrm{f}}$ (displayed in Fig. 6) in which the propagation of a horizontal crack is modeled.

When using a relatively coarse finite element mesh, the force-displacement curve displayed in Fig. 7(a), presents the sawteeth aspect already reported in Refs. [38,39] for smeared approaches.

When the problem is solved with a finer mesh, the response is smoother as can be checked in Fig. 7(b).Therefore, the observed ratcheting in the force-displacement curve is a numerical, and not a physical, effect. However this effect can become unavoidable, as brittle materials are modeled and coarse meshes, limited by the affordable computational cost, are used: typically in three-dimensional problems.

Every teeth of the curve corresponds to the activation (failure) of one or several elements in the crack path, and exhibits limit or turning points. The presence of so many and so close instability points makes the advance of the numerical simulation extremely cumbersome, and time consuming, when standard implicit integration schemes are used.

In Fig. 8 the robustness and the obtained results, for the coarse mesh in terms of the force-displacement curve, are compared when using the standard implicit and the proposed implicit/explicit integration schemes. Also the evolution of the condition number for the extended algorithmic stiffness matrix (considering the additional equation provided by the arc-length restriction) evaluated as the ratio of the minimum and the maximum eigenvalues (inverse of the condition number), is plotted.

When a displacement arc-length control of the increasing vertical displacement at point A, in Fig. 6(a), is used, the analysis with the implicit integration scheme diverges very soon, as it is shown in the upper Fig. 8(a). The divergence time step coincides with the appearance of the first null/negative eigenvalue of the (tangent stiffness) matrix as it is shown in the lower

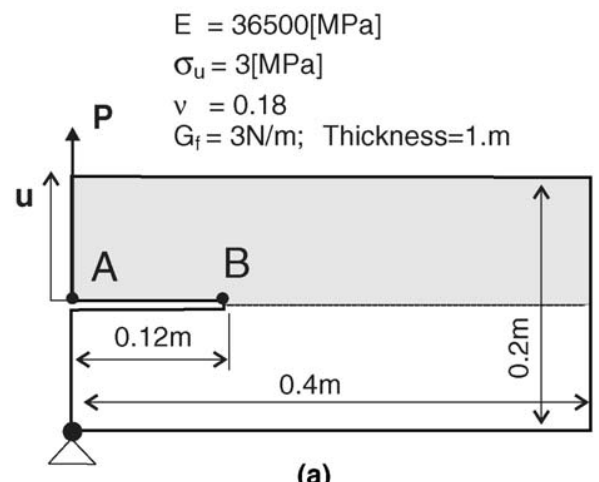

(a)

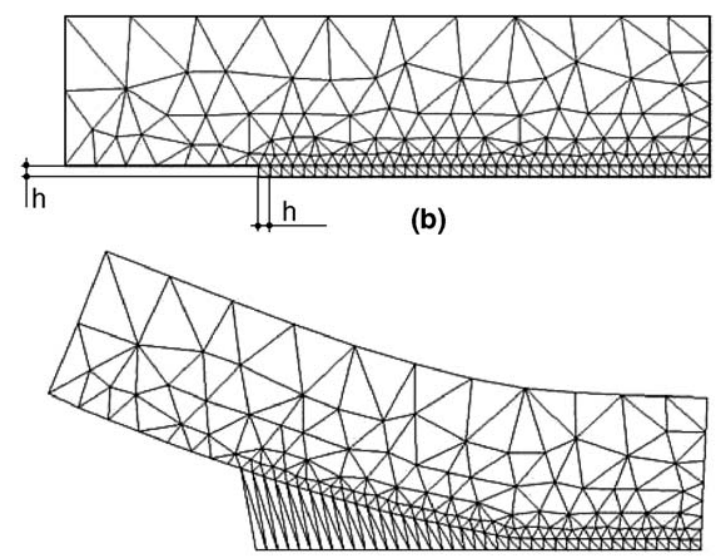

(c)

Fig. 6. DCB test in a concrete specimen: (a) geometrical and material description, (b) finite element mesh (half of the specimen), (c) deformed mesh. 

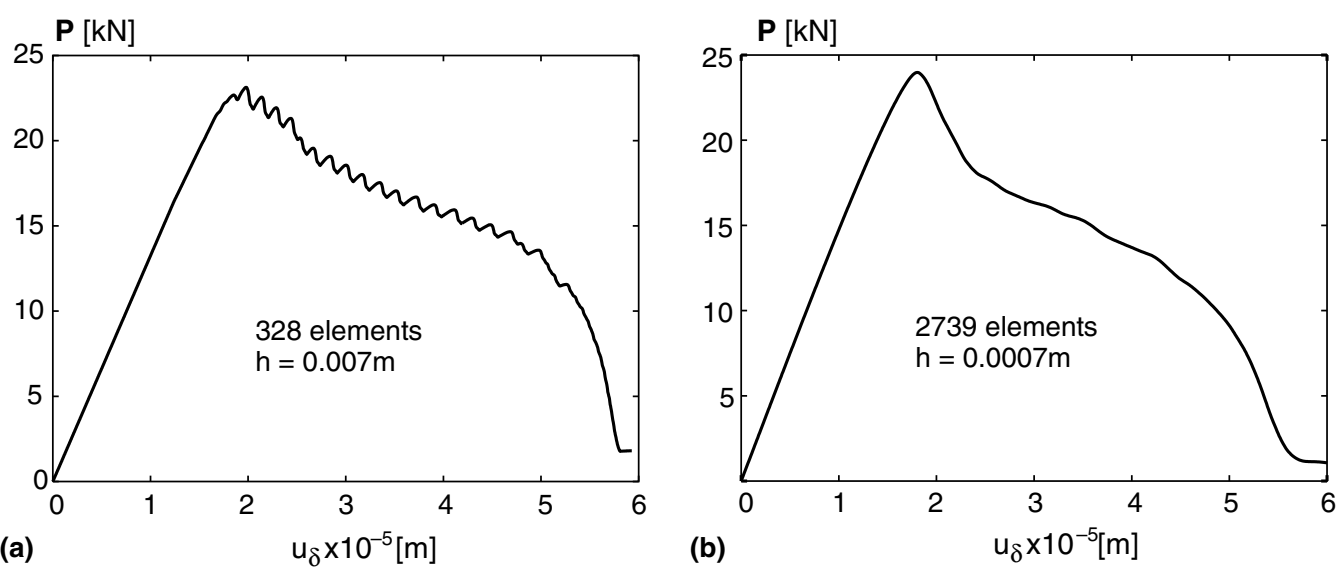

Fig. 7. DCB test in a concrete specimen. Force displacement curves using the implicit/explicit integration procedure: (a) coarse mesh, (b) fine mesh.
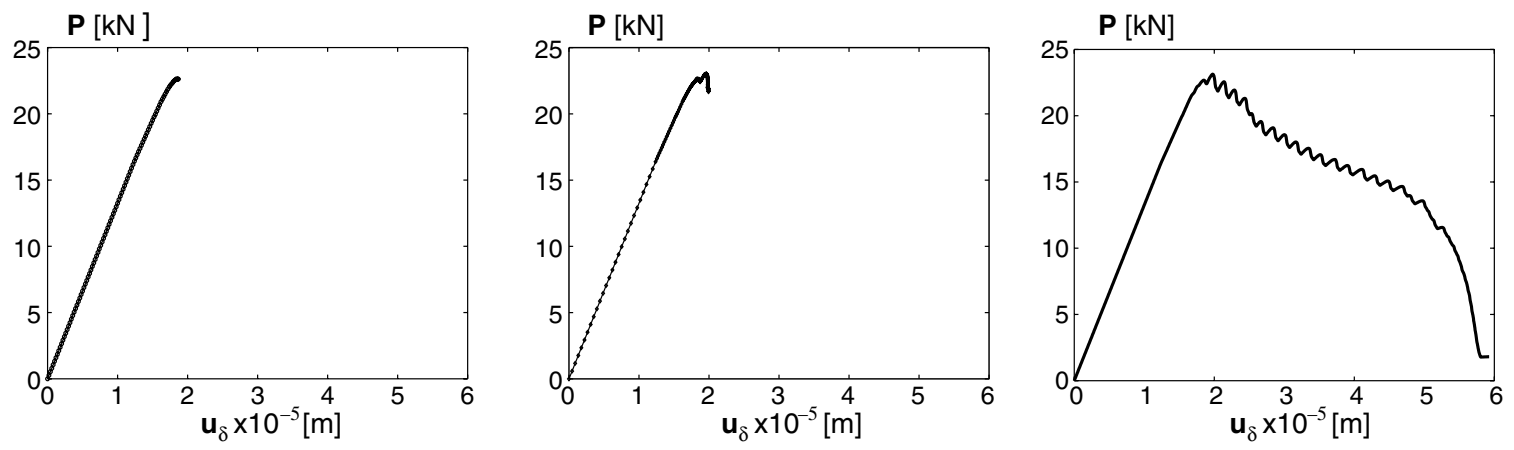

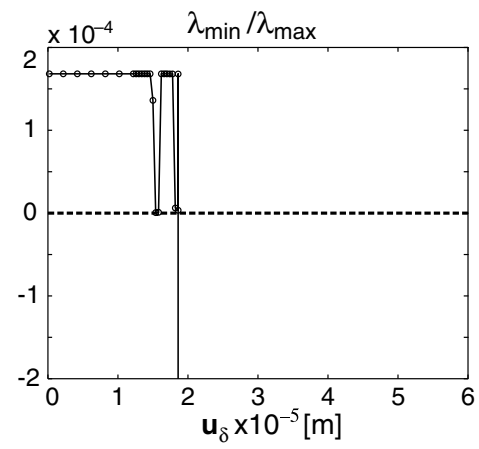

(a)

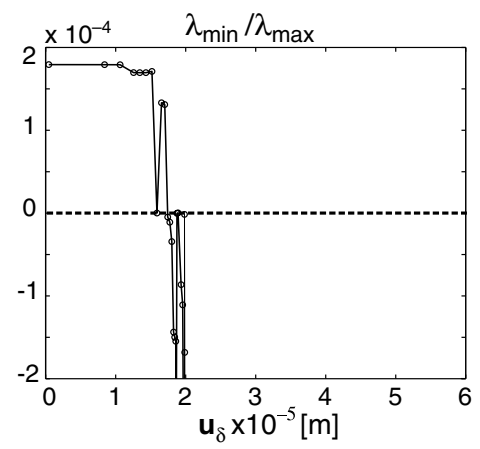

(b)

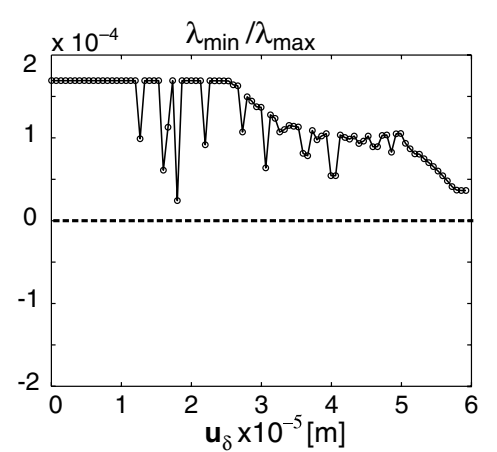

(c)

Fig. 8. DCB test in a concrete specimen. (Up) Force displacement curves, (Down) evolution of the condition number of the extended stiffness matrix. (a) Control of the vertical displacement at point A and implicit integration scheme, (b) control of the vertical displacement at point B and implicit integration scheme, (c) control of the vertical displacement at point A and implicit/explicit integration scheme.

Fig. 8(a). Then, one could think of a smarter continuation algorithm, and perform an arc-length control of the vertical displacement of point B (at the notch tip). In this case, the simulation can be driven a little further, with a constant time step length, but it also diverges very soon after, as it is shown in Fig. 8(b). Again, divergence coincides with the appearance of null/negative eigenvalues of the extended stiffness matrix as it is displayed in the lower Fig. 8(b). More sophisticated time advancing procedures, as an automatic time stepping, could be used but, then, the length of the time step reduces several orders of magnitude, leading to unaffordable computational costs.

However, when the simulation is performed using the proposed implicit/explicit integration scheme of Section 2.4, the analysis can be completed at no difficulty with a constant time step length (with only one iteration per time step) and the complete force-displacement curve of Fig. 8(c) is obtained. In this case, the effective stiffness matrix is always positive definite, as it is shown in the lower Fig. 8(c). All the eigenvalues are positive and the stiffness matrix is far away from being ill conditioned as predicted by the considerations in Section 2.4.3. 


\section{Representative numerical simulations}

The benefits of the proposed combination of a symmetric E-FEM method and the implicit/explicit integration scheme, for purposes of material failure modeling, are essentially of two types:

- Providing robustness to the analysis, and obtaining responses in cases that implicit integration schemes fail.

- Decreasing the computational cost, even in those problems that can be solved with standard implicit integration schemes.

The following examples display these benefits.

\subsection{Tests on the convergence of the implicitlexplicit integration}

The goal of these tests is to evaluate and compare the accuracy, and the required computational cost, when using either the implicit or the implicit/explicit integration schemes. Comparisons are done using a number of structural failure tests, considering both plasticity and continuum damage material models, either in $2 \mathrm{D}$ or 3D problems. The accuracy of the implicit/explicit scheme is evaluated by comparing the solutions, obtained using constant step lengths, without any specific control of the integration error, with those obtained with the purely implicit integration procedure.

\subsubsection{Continuum damage model: four-point bending test on a concrete specimen}

The four-point bending test is a well-known problem that has widely been used in the literature as a numerical benchmark. Here, the experiment reported in [5] is simulated as a 2D problem assuming plane stress conditions. The structure is a notched concrete beam subjected to the load system described in Fig. 9. The geometry and material data are also displayed there. The finite element mesh has approximately 1900 triangular elements.

The material has been modeled using the constitutive damage model in Eqs. (4)-(10) and its parameters are defined in Fig. 9. The experimental solution shows a curved crack, propagating from the notch tip toward the load point D.

In Fig. 9(right) the load versus crack mouth sliding displacement (CMSD) curves, obtained with the implicit integration procedure and three different time step lengths for the implicit/explicit scheme, are displayed. The last three cases were obtained by imposing an incremental CMSD of values $4 \Delta u_{y}, 2 \Delta u_{y}$ and $\Delta u_{y}$, respectively, whereas the implicit solution was obtained with an automatic time step procedure (shortening the time step as the convergence is not achieved in a given number of iterations) imposing the initial value $\Delta u_{y}$.

Table 1 compares the computational times in all cases. Notice that, as expected, the implicit/explicit scheme only requires one iteration per step. From Fig. 9, we also observe a clear convergence, by reducing the time step length, of the implicit/explicit solutions to the purely implicit one. In particular, for the case that the incremental step is fixed to $\Delta u_{y}$, the implicit/explicit integration provides a solution indistinguishable from the implicit one, whereas the required computational cost is five times smaller.

\subsubsection{Elasto-plastic model: slope instability problem}

The slope instability problem taken from [36] is analyzed in this test, by using the elasto-plastic material model in Eqs. (4)-(10). A vertical load is applied to a rigid foot placed on the top of an embankment, as shown in Fig. 10. The geotechnical structure is assumed to respond as a $\mathbf{J} 2$ plasticity material model whose parameters are Young's modulus, $E=10$
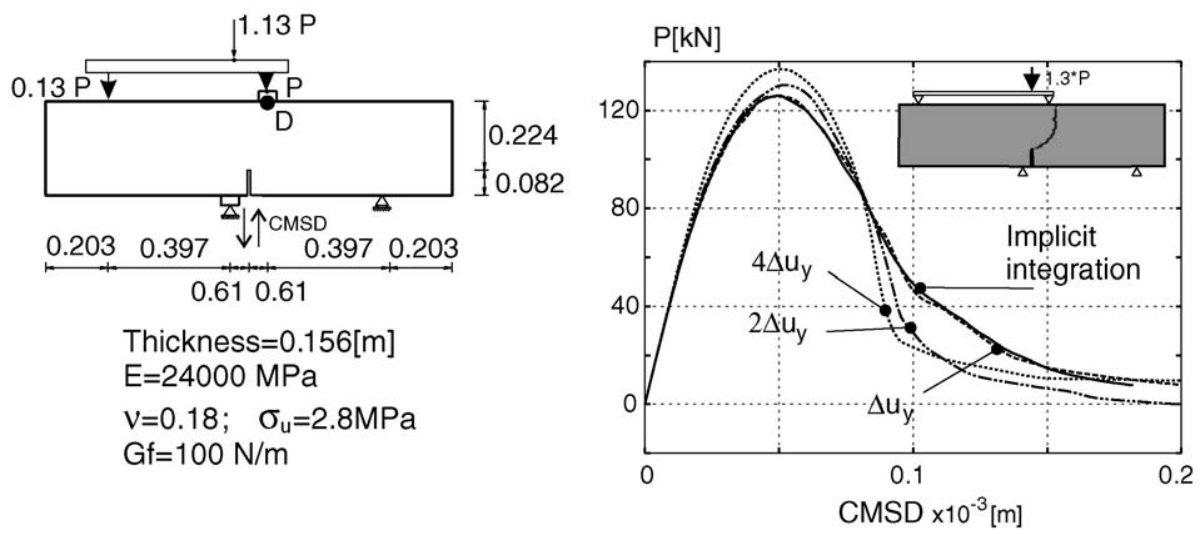

Fig. 9. Four-point bending test. 
Table 1

Four point bending test

\begin{tabular}{llll}
\hline & Steps (total number of iterations) & CPU time $[\mathrm{s}]$ & CPU/CPU (implicit) \\
\hline Implicit/explicit & & & $1 / 16$ \\
$4 \Delta \mathbf{u}_{y}$ & $78(78)$ & 20 & $1 / 10$ \\
$2 \Delta \mathbf{u}_{y}$ & $141(141)$ & 34 & $1 / 5$ \\
$\Delta \mathbf{u}_{y}$ & $260(260)$ & 62 & $1 / 1$ \\
Implicit & $370(\approx 1200)$ tolerance $=1 \mathrm{E}-4$ & 325 & \\
FE mesh: $\approx 1900$ triangles & & &
\end{tabular}

Comparative computational costs using the implicit/explicit and the standard implicit integration schemes.
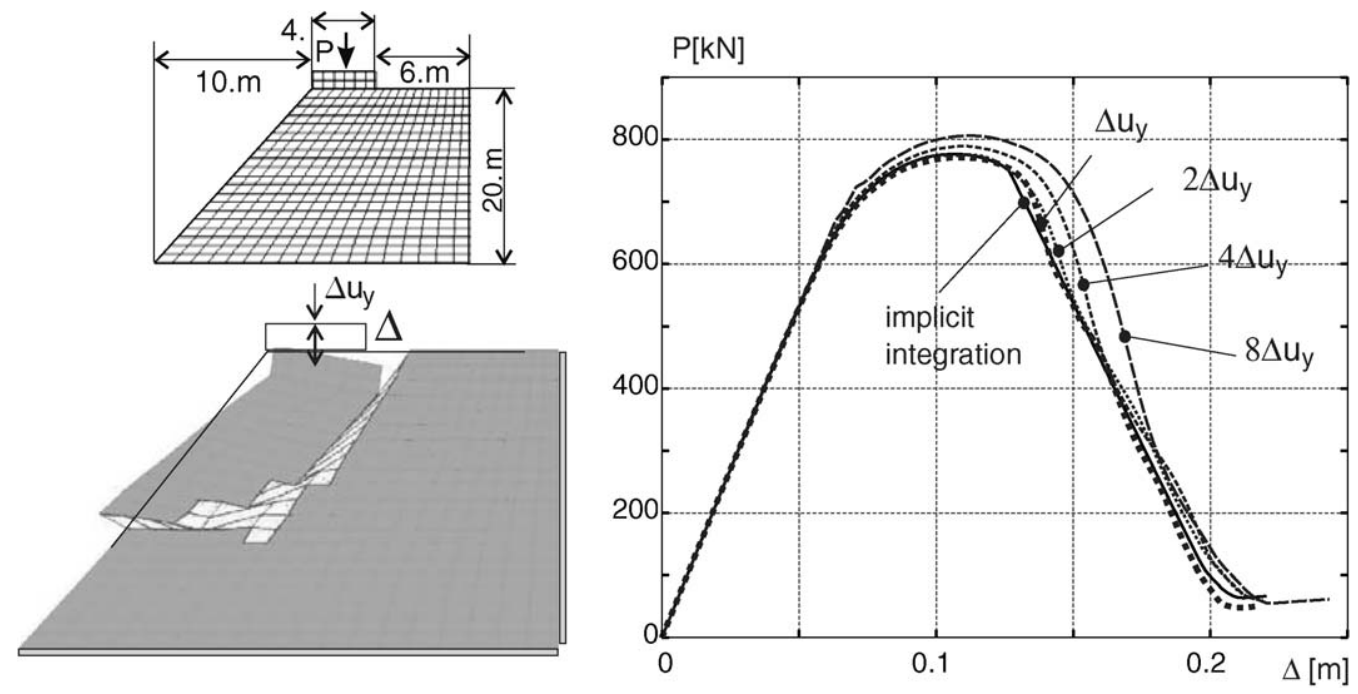

Fig. 10. Slope instability problem.

Table 2

Slope instability problem

\begin{tabular}{lcccc}
\hline & Steps & CPU time [s] (implicit) & CPU time [s] (implicit/explicit integration) & CPU(i)/CPU(i/e) \\
\hline $8 \Delta \mathbf{u}_{y}$ & 75 & 23.9 & 7.8 & 3.06 \\
$4 \Delta \mathbf{u}_{y}$ & 150 & 46.4 & 15.3 & 3.03 \\
$2 \Delta \mathbf{u}_{y}$ & 300 & 86.5 & 30.8 & 2.80 \\
$\Delta \mathbf{u}_{y}$ & 600 & 165.2 & 61.0 & 2.71 \\
FE mesh: 400 quadrilaterals (BBAR) & &
\end{tabular}

Comparative computational cost.

MPa, Poisson's ratio, $v=0.4$, uniaxial peak stress, $\sigma_{\mathrm{u}}=0.1 \mathrm{MPa}$, and intrinsic softening modulus: $\bar{H}=-0.33 \mathrm{MPa} \mathrm{m}^{-1}$. The geometrical data and boundary conditions of the problem are shown in Fig. 10. Plane strain conditions are assumed.

The standard underlying elements are quadrilaterals, enriched with a B-bar technology to account for the locking effects in $\mathrm{J} 2$ plasticity models [42]. The finite element mesh is made of approximately 400 of those finite elements.

Results in all cases have been obtained by controlling the downward displacement component in the rigid foot middle point. Fig. 10 plots the loads vs. vertical displacement curves obtained with the implicit/explicit integration procedure and four different incrementally imposed displacements of values $8 \Delta u_{y}, 4 \Delta u_{y}, 2 \Delta u_{y}$ and $\Delta u_{y}$. These solutions are compared with the ones obtained with the purely implicit integration procedure (which are indistinguishable in the plots for the different considered time step lengths). Again, we can observe the clear convergence of the results from the implicit/explicit integration procedure to the ones obtained with the implicit integration.

The accuracy and robustness exhibited by the implicit integration method in this example (no step shortening was necessary in any case) would not make crucial the use of the implicit/explicit integration method. However, as it is shown in Table 2, the implicit/explicit methods leads, for the same time step length, to substantial reductions in the required CPU time (at the cost of a reduction in the accuracy for large time steps). 


\subsubsection{Continuum damage model: $3 D$ double-notched beam test}

The double-notched concrete beam experiment reported in [7] is also a well-known problem in concrete fracture mechanics simulation. The test, which consists of a concrete beam with two symmetric notches loaded as shown in Fig. 11, has been widely simulated as a $2 \mathrm{D}$ problem (in plane-stress conditions). However, in the present simulation the 3D modeling, a much more challenging problem, in terms of robustness, is considered. Two cracks propagate across the beam, from the notch roots toward the loading points, but only one of them remains in a loading state (active) after the structural peak load.

The solutions in Fig. 11, in terms of the load $\mathbf{F}$ versus CMOD curves, have been obtained using the implicit/explicit integration algorithm, and the symmetrical finite element formulation, in a mesh of 2967 tetrahedra. The load control method consists of imposing a given updated normal plane arc-length procedure of sizes $\Delta s, 2 \Delta s, 4 \Delta s$ and $8 \Delta s$ in each case. With this type of control, convergence of the standard implicit integration scheme fails in all the cases, even using automatic reductions of the time step, before reaching the end of the analysis. This is an example, also observed by the authors in many other cases, of the loss of robustness of the 3D simulations, based on implicit integrations, as compared with the corresponding 2D simulations. On the contrary, for the implicit/explicit procedure, stable analysis, showing a clear convergence with shortening of the incremental displacement control, can be done at no difficulty.

Fig. 12 shows the crack pattern provided by the simulation, exhibiting the two experimentally observed cracks, and the evolution of the cracking across the detected failure surfaces. There, it can also be observed as the crack generated at the upper notch arrests, whereas the other crack progresses determining the final failure mode.
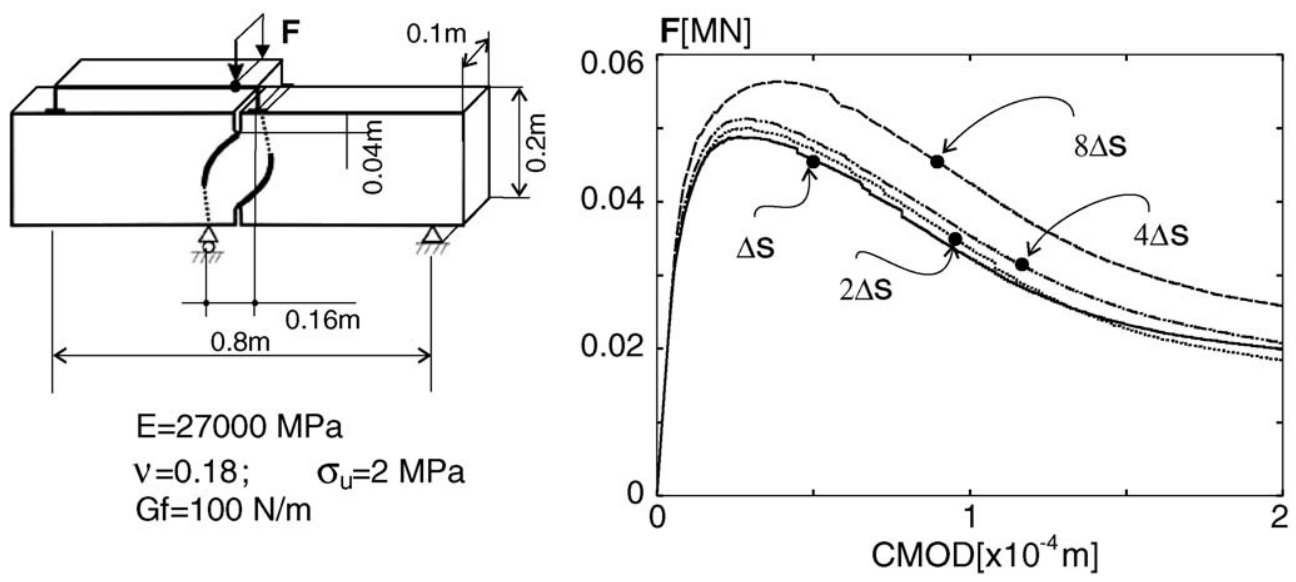

Fig. 11. 3D double-notched concrete beam test.

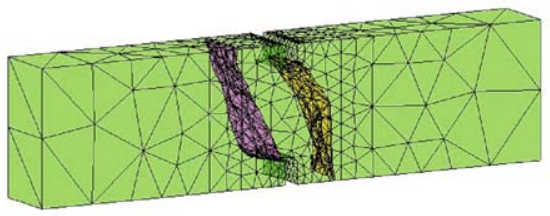

(a)
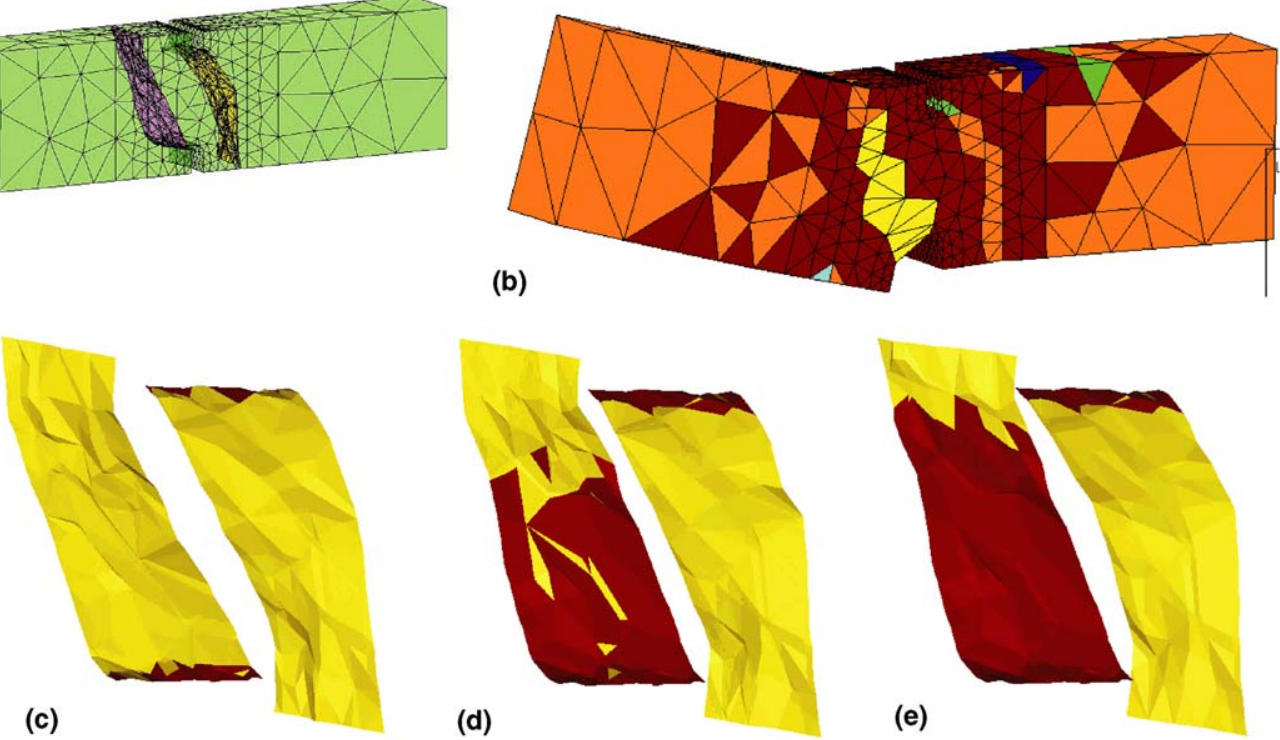

Fig. 12. 3D double-notched beam: (a) numerically obtained failure surfaces (cracks), (b) amplified deformation mode and crack patterns, (c-e) evolution, along time, of damage at the cracking surfaces. 


\subsection{Additional representative $3 D$ simulations}

In order to illustrate the potential of the CSDA in modelling realistic structural failure mechanisms, applications of the preceding methodology to several three dimensional failure problems are presented next. Both continuum constitutive models of Section 1.1 (continuum damage and elasto-plasticity) are considered to represent failure. In all the cases, the symmetric finite element with elemental enrichment of Section 2.3 and the implicit/explicit integration method of Section 2.4 have been used.

\subsubsection{Brazilian test}

The splitting test in a cylindrical specimen (Brazilian test, see [15]), compressed along two diametrically opposed lines, as shown in Fig. 13, is modelled using the continuum damage model. The expected failure surface is a diametric vertical crack plane containing the two load lines. Fig. 13(right) shows the load vs. transversal deformation curve, scaled in terms of the peak load $\left(P_{\max }\right)$. The load decreases after the peak (up to approximately $80 \%$ of $P_{\max }$ ), due to the complete propagation of the primary crack system, which compares well with the experimental results in [15]. The analysis was stopped at the subsequent raising branch as the two split halves start resisting in compression.

Also in the curve in Fig. 13(right) the propagation of the cracking during the load process is displayed. This exemplifies the potential of three-dimensional simulations, as the one presented here, as providers of unusual and useful information about the failure propagation mechanisms (from the exterior to the interior and from the centre to the loading lines as it is shown in the figure).

Fig. 14 shows the considered finite element mesh of tetrahedra, and the (amplified) deformed shape of the specimen at the end of the analysis.

\subsubsection{Pull out of a bar anchored to a concrete specimen}

The extraction of a steel rod (assumed elastic) anchored to a cylindrical mass of concrete, is modeled. The reference experimental test is that presented in $[13,14]$. Numerical results of a similar test have previously been presented using a 2D model with the axial-symmetric assumption [37]. Here a complete 3D analysis is performed.
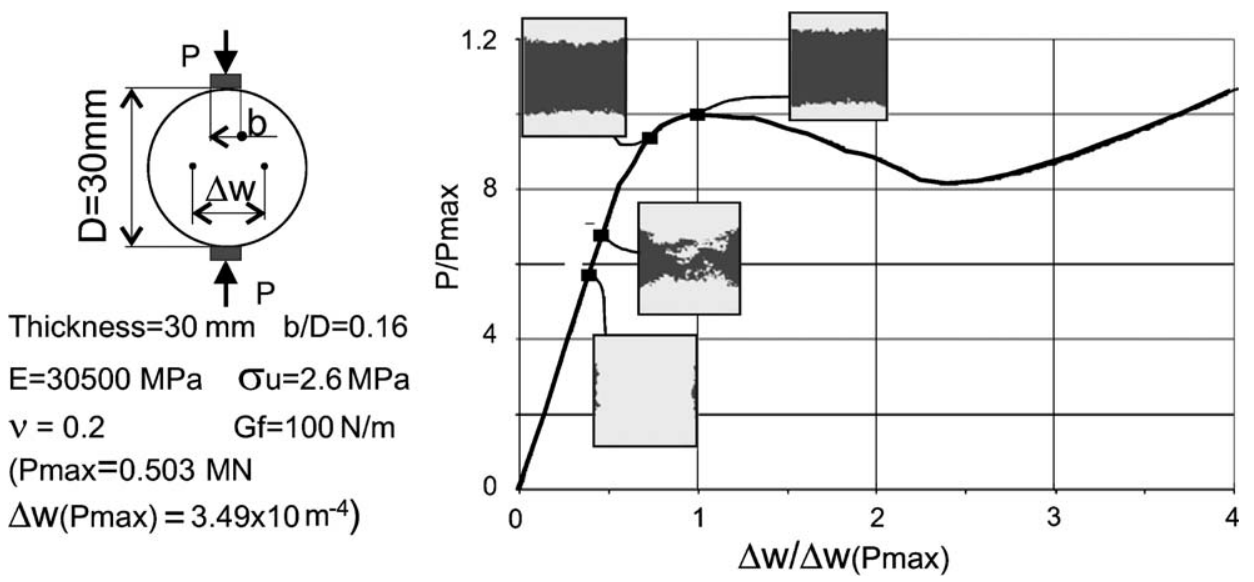

Fig. 13. Splitting test in a cylindrical specimen: material data and load vs. transversal deformation curve.
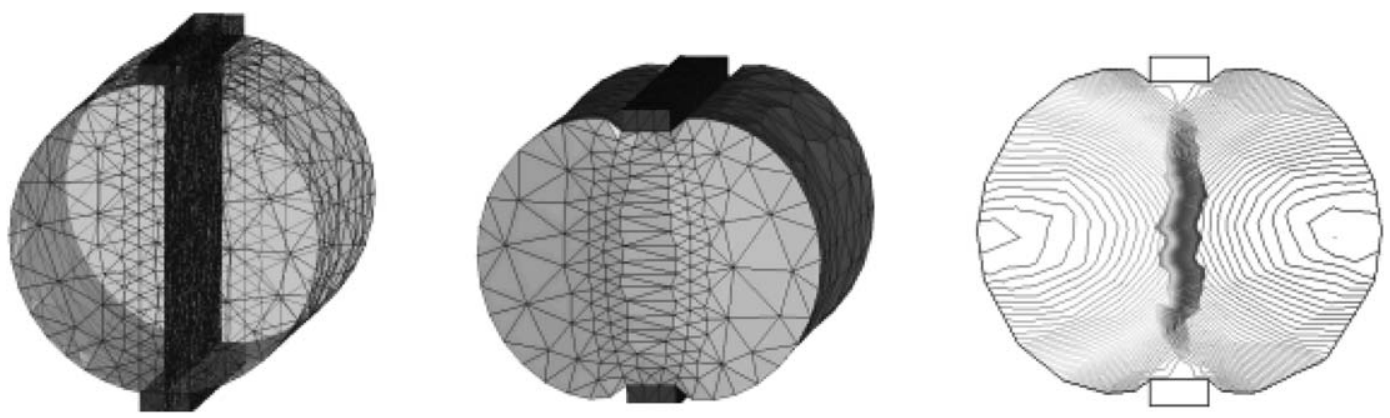

Fig. 14. Splitting test: Finite element mesh, deformed mesh and displacement contours. 
The material properties of the damage model relative to the concrete bulk, are $E=29,000 \mathrm{MPa}, \sigma_{\mathrm{u}}=3.1 \mathrm{MPa}, v=0.2$ and $G_{\mathrm{f}}=150 \mathrm{~N} / \mathrm{m}$. The dimensions of the specimen are shown in Fig. 15 together with the obtained load versus displacement curves (Table 3). The tetrahedral finite element mesh and the typical conical failure surface obtained from the numerical simulations are presented in Fig. 16.
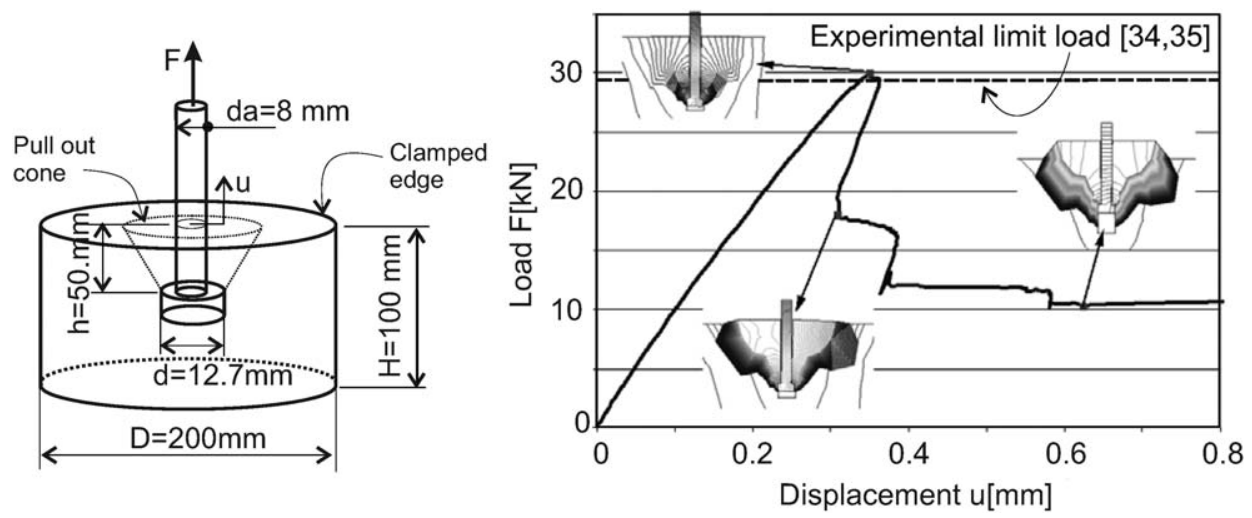

Fig. 15. Pull out of a bar anchored to a concrete specimen: (Left) problem description, (Right) load vs. displacement curve.

Table 3

3D double-notched beam test

\begin{tabular}{|c|c|c|}
\hline Implicit/explicit & Steps & CPU time $[\mathrm{s}][15]$ \\
\hline $8 \Delta s$ & 450 & 764 \\
\hline $4 \Delta s$ & 900 & 1577 \\
\hline $2 \Delta s$ & 1800 & 2943 \\
\hline Implicit & Not $\mathrm{cc}$ & \\
\hline
\end{tabular}

Computational cost using the implicit/explicit integration algorithm (2967 tetrahedra, 2580 d.o.f.).

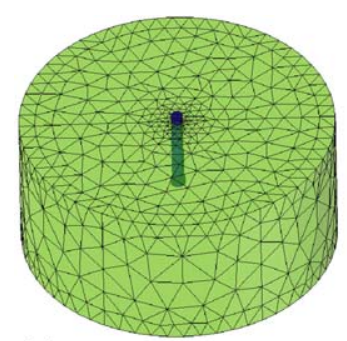

(a)

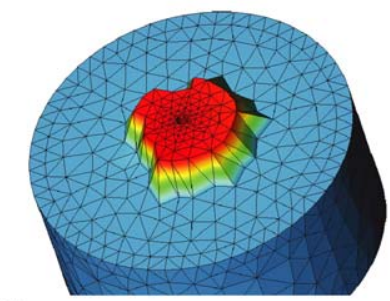

(d)

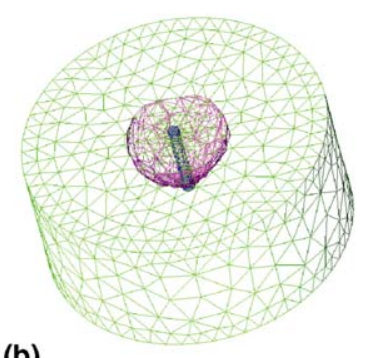

(b)

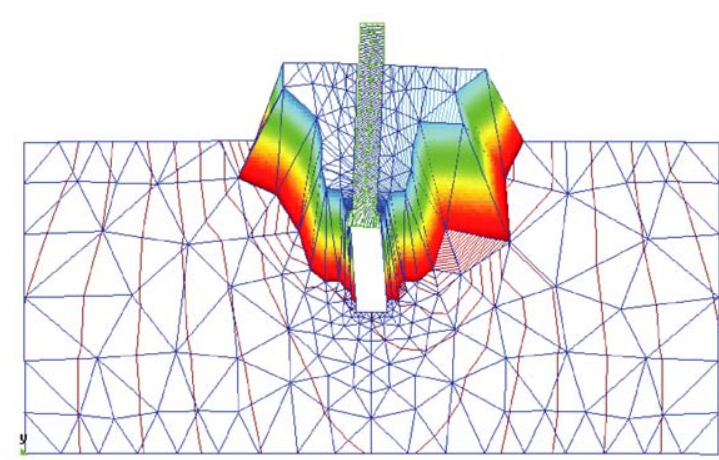

(e)

Fig. 16. Pull out of a bar anchored to a concrete specimen: (a) finite element mesh, (b, c) numerically obtained failure (cracking) surface, (d) external view of the (amplified) deformation pattern showing the localized elements, (e) internal view (cross-section) of the deformation pattern together with the contours of the displacement field. 


\subsubsection{Collapse analysis of a concrete dam}

The structural collapse of a gravity-arch concrete dam is modelled. The geometry of the concrete dam has been taken from [20]. The analysis is done on the solid-foundation domain shown in Fig. 17, where a reduced part of the foundation (rock) is considered. The material properties for the concrete correspond to an artificially made brittle material, in order to induce a clear failure mode that tests the ability of the CSDA to reproduce it. Both, the concrete dam and the rock foundation, have been modeled by the damage model in Eqs. (4)-(10) but now considering a limited strength in both tension and compression regimes. The material properties are displayed in Table 4.

The final goal of the analysis is to reproduce the structural collapse mode and to determine the theoretical safety factor in front of the classical load action on the dam: the hydrostatic loading acting on the up-stream face. Therefore, the typical triangular pressure distribution has been affected by a load factor evolving along the pseudo-time (here the central lateral displacement at the crest). Lateral surfaces, marked as E1 and E2 in Fig. 17(b), and the bottom of the foundation, are assumed clamped. A global tracking procedure, allowing for multiples failure surfaces has been considered [28].

Fig. 17(c) shows the load factor vs. the horizontal displacement at the crest (point P in Fig. 17(a)). The peak load determines the safety factor (here around 3.0) and the final failure mode displays the formation of three large vertical cracks at points $\mathrm{A}, \mathrm{B}$ and $\mathrm{C}$ of the loading process.

\subsubsection{Landslide simulation}

The problem of a landslide modeling through the CSDA is considered here. A soft layer of soil is assumed to rest on the hard bedrock and its collapse, under increasing values of the soil density, through formation of a slip surface, is simulated by considering a $\mathbf{J} 2$ plasticity model. The geometry and material data are shown in Fig. 18. In order to overcome the stresslocking problems associated to J2 plasticity models, the basic element is the hexahedron modified according to the B-bar formulation [42].

The obtained, three-dimensional, collapse mode is presented in Fig. 19. There can be checked that the typical slip surface, observed in landslides (pure slip mode), is appropriately reproduced by the CSDA.

In Fig. 18(right), additional details of the simulation are presented. The load factor vs. displacement curve is plotted together with the corresponding evolution, at different points of the loading process, of the material failure at the slip surface.
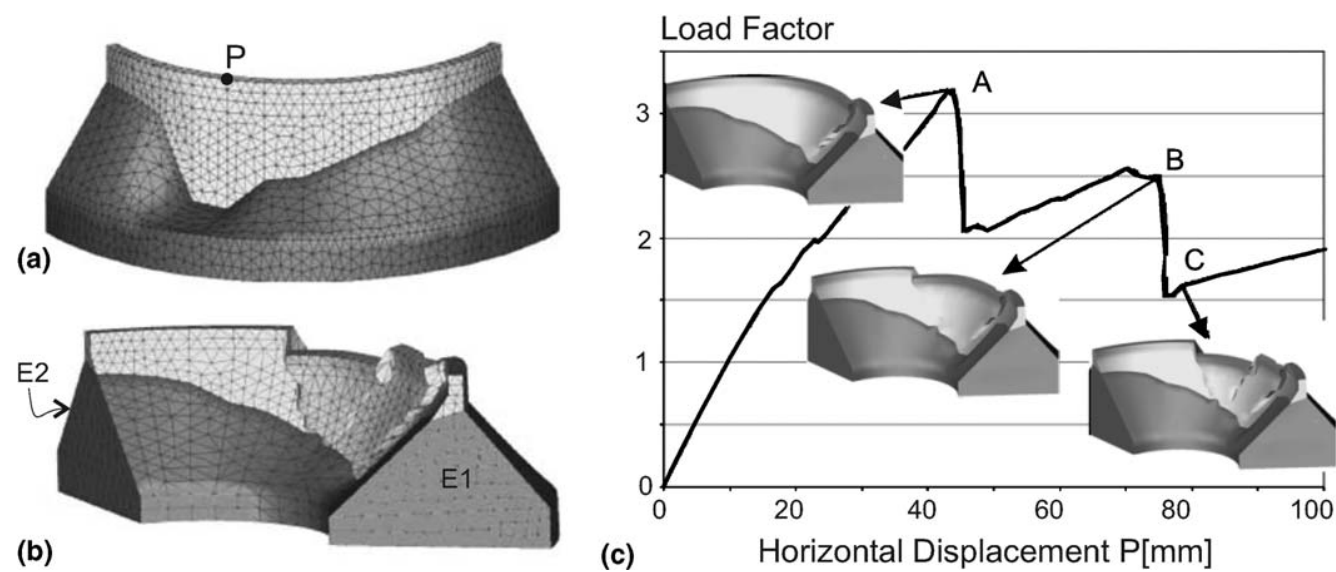

Fig. 17. Collapse analysis of a concrete dam: (a) finite element mesh, (b) final deformed (amplified) mesh showing the collapse mode, (c) load factor vs. horizontal displacement at point $\mathrm{P}$.

Table 4

Dam analysis: mechanical parameters of concrete and rock

\begin{tabular}{lll}
\hline & Concrete & Rock foundation \\
\hline Elastic modulus & $20,000 \mathrm{MPa}$ & $15,000 \mathrm{MPa}$ \\
Mass density & $2300 \mathrm{~kg} / \mathrm{m}^{3}$ & - \\
Poisson coefficient & 0.2 & 0.2 \\
Compressive strength & $11.6 \mathrm{MPa}$ & $10.0 \mathrm{MPa}$ \\
Tensile strength & $1.0 \mathrm{MPa}$ & $1.0 \mathrm{MPa}$ \\
Fracture energy & $100 \mathrm{~N} / \mathrm{m}$ & $100 \mathrm{~N} / \mathrm{m}$ \\
\hline
\end{tabular}




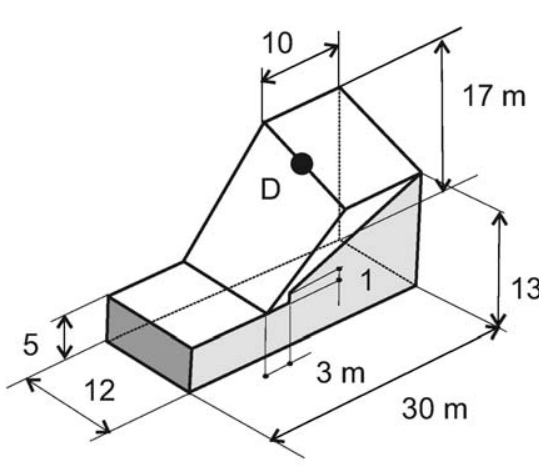

Soil:

$$
\begin{array}{ll}
E=100 \mathrm{MPa} ; & \sigma_{u}=1 \mathrm{MPa} \\
v=0.4 ; & \mathrm{H}=-2 \mathrm{MPa} \\
\bar{H}=-5 \mathrm{MPa} / \mathrm{m} ; & \text { Density }=18 \mathrm{kN} / \mathrm{m}^{3}
\end{array}
$$

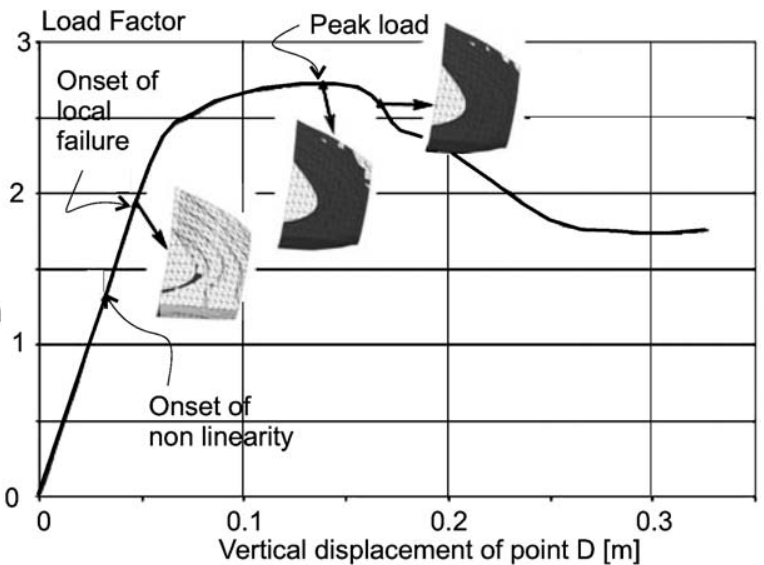

Bedrock:

$$
\begin{aligned}
& E=1000 M P a ; \quad \sigma u=\infty \\
& v=0.4 ; \quad \text { Density }=18 \mathrm{kN} / \mathrm{m}^{3}
\end{aligned}
$$

Fig. 18. Landslide: load factor versus vertical displacement and discontinuity surface propagation.

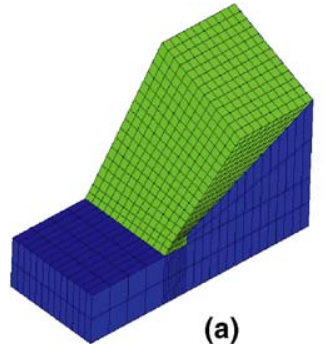

(a)

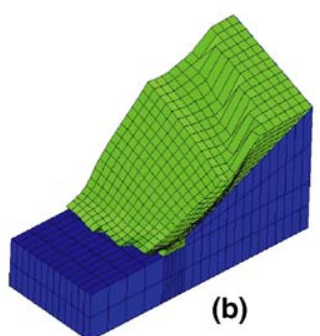

(b)

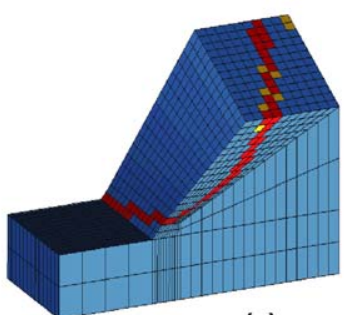

(c)

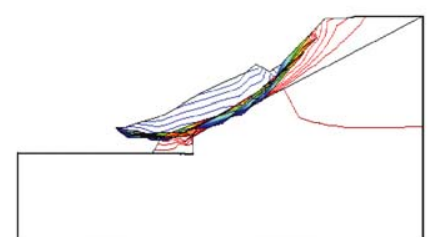

(d)

Fig. 19. Landslide: (a) finite element mesh, (b) deformed (amplified) finite element mesh showing the obtained collapse mode, (c) external view of the detected (slip) failure surface, (d) internal view (cross-section) of the deformation pattern and contours of the displacement field.

\subsection{Computational costs of $3 D$ simulations using the CSDA}

For the simulations presented in the preceding sections Table 5 displays the computational costs based on a single Pen-

\begin{tabular}{|c|c|c|c|c|}
\hline Section (problem) & Number of DOF & Number of elements & Number of time steps & CPU time $[\mathrm{s}]$ \\
\hline 3.2 .2 & 11,505 & 18,872 (tetrahedra) & 89 & 7154 \\
\hline 3.2 .4 & 12,264 & 3354 (hexahedra) & 90 & 2016 \\
\hline
\end{tabular}
tium $^{\circledR} 4$ processor, at $3.2 \mathrm{GHz}$, and $1 \mathrm{~Gb}$. of RAM memory. As it can be observed there, the computational costs, for the different simulations, are very affordable and range from less then one hour to a maximum of about $3 \mathrm{~h}$ of $\mathrm{CPU}$.

Table 5

Computational cost of the 3D simulations 


\section{Concluding remarks}

Throughout the preceding sections, some fundamental issues about the robustness and stability of the Continuum Strong Discontinuity Approach (CSDA) to material failure have been addressed. After identification of lack of symmetry of the finite element formulation, and the material softening in the constitutive model, as possible causes of lack of robustness, a remedy has been proposed as the combination of two specific ingredients in the numerical modeling. The first one is the use of a symmetric version of the (variationally consistent and elementary enriched) finite element with embedded discontinuities E-FEM. From the authors' experience this provides larger robustness when compared with unsymmetrical formulations.

However this seems not to be enough when brittle materials, as concrete, and relatively coarse meshes are used. The problem becomes crucial in large scale (three-dimensional) computations when the number of elements is very limited by the affordable computational cost. The second proposed ingredient is an implicit-explicit integration procedure of the continuum constitutive model that leads to the so-called effective algorithmic operator, as the corresponding tangent constitutive operator, which is constant and positive definite.

The use of these procedures guaranties the positive definite character and the constancy of the algorithmic stiffness of the problem during every time step, and provides a substantial increase of the robustness of the simulation, as well as a dramatic reduction of the required computational time. In compensation, some reduction on the accuracy can be expected although convergence to the exact solution with the reduction of the length of the time step has been checked. As it is shown in the presented examples, the proposed methodology allows tackling three-dimensional simulations of material failure in small computers in very affordable times.

\section{Acknowledgements}

Financial support from the Spanish Ministry of Science and Technology, through grants BIA2004-07289-C03-02 and BIA2004-02080, and from the Catalan Government Research Department, through the CIRIT grant 2001-SGR 00262, is gratefully acknowledged.

\section{Appendix A}

Let us consider the matrix $\mathbf{K}$ obtained by assembling of the elemental matrices $\mathbf{K}^{(e)}$ defined as

$$
\mathbf{K}=\mathscr{A}\left(\mathbf{K}^{(e)}\right), \quad \mathbf{K}^{(e)}=\int_{\Omega^{(e)}} \mathbf{B}^{(e)^{\mathrm{T}}} \cdot \mathbf{C}^{\mathrm{alg}} \cdot \mathbf{B}^{(e)} \mathrm{d} \Omega,
$$

where the matrix $\mathbf{C}^{\text {alg }}(\mathbf{x})$ is positive definite, therefore: $\mathbf{w}^{\mathrm{T}} \cdot \mathbf{C}^{\mathrm{alg}} \cdot \mathbf{w}>0 \forall \mathbf{w} \neq \mathbf{0} \forall \mathbf{x}$. Then,

$$
\mathbf{v}^{\mathrm{T}} \cdot \mathbf{K} \cdot \mathbf{v}=\sum_{e=1}^{n_{\mathrm{elem}}} \mathbf{v}^{(e)^{\mathrm{T}}} \cdot \mathbf{K}^{(e)} \cdot \mathbf{v}^{(e)}=\sum_{e=1}^{n_{\mathrm{elem}}} \int_{\Omega^{(e)}} \underbrace{\mathbf{v}^{(e)^{\mathrm{T}}} \cdot \mathbf{B}^{(e)^{\mathrm{T}}}}_{\mathbf{w}^{\mathrm{T}}} \cdot \mathbf{C}^{\mathrm{alg}} \cdot \underbrace{\mathbf{B}^{(e)} \cdot \mathbf{v}^{(e)}}_{\mathbf{w}} \cdot \mathrm{d} \Omega=\sum_{e=1}^{n_{\mathrm{elem}}} \int_{\Omega^{(e)}} \underbrace{\mathbf{w}^{\mathrm{T}} \cdot \mathbf{C}^{\mathrm{alg}} \cdot \mathbf{w}}_{>0} \cdot \mathrm{d} \Omega>0 \quad \forall \mathbf{v} \neq 0
$$

and matrix $\mathbf{K}$ is positive definite.

\section{References}

[1] J. Alfaiate, New developments in the study of strong embedded discontinuities in finite elements, Adv. Fract. Damage Mech. 251-2 (2003) 109-114.

[2] J. Alfaiate, A. Simone, L.J. Sluys, Non-homogeneous displacement jumps in strong embedded discontinuities, Int. J. Solids Struct. 40 (2003) 5799 5817.

[3] F. Armero, K. Garikipati, Recent advances in the analysis and numerical simulation of strain localization in inelastic solids, Presented at Computational Plasticity. Fundamentals and Applications (1995).

[4] F. Armero, K. Garikipati, An analysis of strong discontinuities in multiplicative finite strain plasticity and their relation with the numerical simulation of strain localization in solids, Int. J. Solids Struct. 33 (1996) 2863-2885.

[5] M. Arrea, A.R. Ingraffea, Mixed-mode Crack Propagation in Mortar and Concrete, Dept. Struct. Eng. cornell Univ., New York, 1982 , pp. 81-13.

[6] T. Belytschko, N. Möes, S. Usui, C. Parimi, Arbitrary discontinuities in finite elements, Int. J. Numer. Methods Engrg. 50 (2001) $993-1013$.

[7] P. Bocca, A. Carpintieri, S. Valente, Size effect in the mixed mode crack propagation: softening and snap-back analysis, Engrg. Fract. Mech. 35 (1990) $159-170$.

[8] G. Bolzon, A. Corigliano, A discrete formulation for elastic solids with damaging interfaces, Comput. Methods Appl. Mech. Engrg. 140 (1997) 329359.

[9] G. Bolzon, A. Corigliano, Finite elements with embedded displacement discontinuity: a generalized variable formulation, Int. J. Numer. Methods Engrg. 49 (2000) 1227-1266.

[10] C.C. Celigoj, On strong discontinuities in anelastic solids, A finite element approach taking a frame indifferent gradient of the discontinuous displacements, Presented at Int. J. Numer. Meth. Engng., 2000.

[11] R. de Borst, G.N. Wells, L.J. Sluys, Some observations on embedded discontinuity models, Engrg. Comput. 18 (2001) $241-254$. 
[12] E.N. Dvorkin, A.M. Cuitino, G. Gioia, Finite elements with displacement embedded localization lines insensitive to mesh size and distortions, Int. J. Numer. Methods Engrg. 30 (1990) 541-564.

[13] R. Eligehausen, J. Ozbolt, Size effect in design of fastenings, in Mechanics of quasi-brittle materials and structures a volume in honour of prof. Zdenek P. Bazant 60th birthday: Gilles Pijaudier-Cabot, Zdenek Bittnar, Bruno Gérard, Paris Editions Hermes, 1999, pp. 95-118.

[14] R.B.P.C.V. Eligehausen, R. Pukl, Size effect of the concrete cone failure load of anchor bolts, Presented at Framcos-1, 1992.

[15] G.V. Guinea, M. Elices, J. Planas, Measuring the tensile strength through size effect curves, Presented at Fracture Mechanics of Concrete Strucutres, Framcos-3, Gifu, Japan, 1998.

[16] M. Jirasek, Comparative study on finite elements with embedded discontinuities, Comput. Methods Appl. Mech. Engrg. 188 (2000) $307-330$.

[17] R. Larsson, K. Runesson, N.S. Ottosen, Discontinuous displacement approximation for capturing plastic localization, Int. J. Numer. Methods Engrg. 36 (1993) 2087-2105.

[18] H.R. Lofti, P.B. Shing, Embedded representation of fracture in concrete with mixed finite-elements, Int. J. Numer. Methods Engrg. 38 (1995) 13071325.

[19] N. Moës, N. Sukumar, B. Moran, T. Belytschko, An extended finite element method (X-FEM) for two and three-dimensional crack modelling. Presented at ECCOMAS 2000, Barcelona, Spain, 2000.

[20] NW-IALAD. Integrity Assessment of large concrete dams, European Research Network. Available from: < http://nw-ialad.uibk.ac.at/Wp2/Tg3/Se5/ Ss $9 />$.

[21] J. Oliver, A consistent characteristic length for smeared cracking models, Int. J. Numer. Methods Engrg. 28 (1989) $461-474$.

[22] J. Oliver, Continuum modelling of strong discontinuities in solid mechanics using damage models, Comput. Mech. 17 (1995) $49-61$.

[23] J. Oliver, Modelling strong discontinuities in solid mechanics via strain softening constitutive equations. 1 Fundamentals, Int. J. Numer. Methods Engrg. 39 (1996) 3575-3600.

[24] J. Oliver, Modelling strong discontinuities in solid mechanics via strain softening constitutive equations. 2. Numerical simulation, Int. J. Numer. Methods Engrg. 39 (1996) 3601-3623.

[25] J. Oliver, On the discrete constitutive models induced by strong discontinuity kinematics and continuum constitutive equations, Int. J. Solids Struct. 37 (2000) 7207-7229.

[26] J. Oliver, Topics on failure mechanics, International Center for Numerical Methods in Engineering (CIMNE) Monograph no. 68 (2002).

[27] J. Oliver, M. Cervera, O. Manzoli, Strong discontinuities and continuum plasticity models: the strong discontinuity approach, Int. J. Plast. 15 (1999) $319-351$.

[28] J. Oliver, A.E. Huespe, Continuum approach to material failure in strong discontinuity settings, Comput. Methods Appl. Mech. Engrg. 193 (2004) $3195-3220$.

[29] J. Oliver, A.E. Huespe, Theoretical and computational issues in modelling material failure in strong discontinuity scenarios, Comput. Methods Appl. Mech. Engrg. 193 (2004) 2987-3014.

[30] J. Oliver, A.E. Huespe, M.D.G. Pulido, E. Chaves, From continuum mechanics to fracture mechanics: the strong discontinuity approach, Engrg. Fract. Mech. 69 (2002) 113-136.

[31] J. Oliver, A.E. Huespe, M.D.G. Pulido, E. Samaniego, On the strong discontinuity approach in finite deformation settings, Int. J. Numer. Methods Engrg. 56 (2003) 1051-1082.

[32] J. Oliver, A.E. Huespe, E. Samaniego, A study on finite elements for capturing strong discontinuities, Int. J. Numer. Methods Engrg. 56 (2003) $2135-$ 2161.

[33] J. Oliver, A.E. Huespe, E. Samaniego, E.W.V. Chaves, Continuum approach to the numerical simulation of material failure in concrete, Int. J. Numer. Anal. Methods Geomech. 28 (2004) 609-632.

[34] J. Oliver, A.E. Huespe, P.J. Sánchez, A comparative study on finite elements with embedded discontinuities: E-FEM vs. X-FEM, Comput. Methods Appl. Mech. Engrg., in press.

[35] R.L. Borja, R.A. Regueiro, A finite element model for strain localization analysis of strongly discontinuous fields based on standard Galerkin approximation, Comput. Methods Appl. Mech. Engrg. (2000) 1529-1549.

[36] R.A. Regueiro, R.I. Borja, Plane strain finite element analysis of presure sensitive plasticity with strong discontinuity, Int. J. Solids Struct. (2001) 3647-3672.

[37] J.G. Rots, Computational Modeling of Concrete Fractures, Delft University of Technology, 1988.

[38] J.G. Rots, Sequentially linear continuum model for concrete fracture, in: R. de Borst, J. Mazars, G. Pijaudier-Cabot, A.A.J.G.M. van Mier (Eds.), Fracture Mechanics of Concrete Structures, Balkema, 2001, pp. 831-839.

[39] J.G. Rots, S. Invernizzi, Regularized saw-tooth softening, in: N. Bicanic, R.D. Borst, H. Mang, G. Meschke (Eds.), Computational Modelling of Concrete Structures, A.A. Balkema, The Netherlands, 2003, pp. 599-617.

[40] J. Simo, J. Oliver, A new approach to the analysis and simulation of strong discontinuities, presented at Fracture and Damage in Quasi-brittle Structures, 1994.

[41] J. Simo, J. Oliver, F. Armero, An analysis of strong discontinuities induced by strain-softening in rate-independent inelastic solids, Comput. Mech. 12 (1993) 277-296.

[42] J.C. Simo, T.J.R. Hughes, Computational Inelasticity, Springer, 1998. 